\title{
Kepler, Newton and numerical analysis
}

\author{
G. Wanner \\ University of Geneva, \\ Section de Mathématiques, \\ CP 64, CH-1211 Genève 4, \\ Switzerland \\ E-mail: Gerhard.Wanner@unige.ch
}

\begin{abstract}
Numerical methods are usually constructed for solving mathematical problems such as differential equations or optimization problems. In this contribution we discuss the fact that numerical methods, applied inversely, were also important in establishing these models. We show in detail the discovery of the laws of planetary motion by Kepler and Newton, which stood at the beginning of modern science. The 400th anniversary of the publication of Kepler's laws (1609) is a good occasion for this investigation.
\end{abstract}

\section{CONTENTS}

1 Origins of numerical analysis $\quad 561$

2 'Inverse' numerical methods $\quad 567$

3 The origin of Kepler's laws 572

4 Newton's discovery of the Law of Gravitation 584

5 Richard Feynman's elegant proof 592

References $\quad 596$

\section{Origins of numerical analysis}

We start with an overview of the origins of numerical methods for ODEs. The problem we choose is the movement of a body with negligible mass in the gravitational field of two fixed bodies with masses $A$ and $B$, positions $x_{1}=0$ for the first, $x_{1}=a$ for the second, and $x_{2}=0$ for both. The so-called Newton's equations for this problem are

$$
\begin{gathered}
\frac{\mathrm{dd} x_{1}}{\mathrm{~d} t^{2}}=-\frac{A x_{1}}{v^{3}}-\frac{B\left(x_{1}-a\right)}{u^{3}}, \quad \frac{\mathrm{dd} x_{2}}{\mathrm{~d} t^{2}}=-\frac{A x_{2}}{v^{3}}-\frac{B x_{2}}{u^{3}}, \\
v=\sqrt{x_{1}^{2}+x_{2}^{2}}, \quad u=\sqrt{\left(x_{1}-a\right)^{2}+x_{2}^{2}}, \quad A=2, \quad B=1, \quad a=1 .
\end{gathered}
$$


This is one of the problems for which Euler managed, by an incredible tour de force, to find analytical formulas for the solutions in the two-dimensional case (Euler E301 1766) and the three-dimensional case (Euler E328 1767). Here we use this problem to demonstrate numerical methods for the chosen initial position $x_{10}=1.47, \dot{x}_{10}=-0.81, x_{20}=0.8, \dot{x}_{20}=0$.

\subsection{Euler's method}
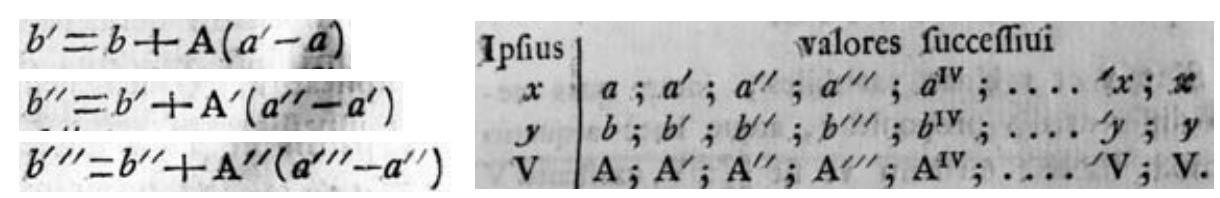

Figure 1.1. First publication of Euler's method in E342, written for the differential equation $\frac{\mathrm{d} y}{\mathrm{~d} x}=V(x, y)$ with initial values $y(a)=b$.

In his monumental three-volume treatise Institutiones Calculi Integralis (Euler E342 1768, E366 1769, E385 1770), after having explained analytic formulas for integrals, so-called Riemann sums for integrals without analytic solutions, and analytic formulas for ordinary differential equations, Euler reached the problem of finding approximate solutions of differential equations without analytic solutions by an extension of the idea of Riemann sums (Euler E342 1768, Pars II, §650). This led to Euler's method, whose first publication, written for the differential equation $\frac{\mathrm{d} y}{\mathrm{~d} x}=V(x, y)$ with initial values $y(a)=b$, is reproduced in Figure 1.1.

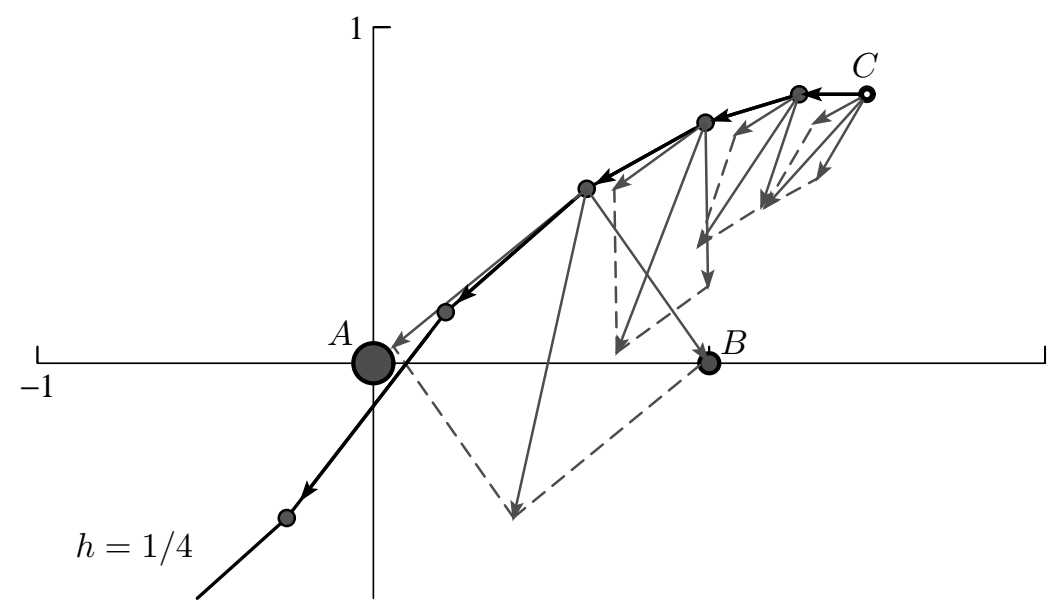

Figure 1.2. The problem with two fixed mass points and solution using Euler's method for $h=1 / 4$. 
For an equation of the type (1.1), i.e.,

$$
\frac{\mathrm{dd} x}{\mathrm{~d} t^{2}}=F(t, x) \quad \text { or } \quad \frac{\mathrm{d} x}{\mathrm{~d} t}=v, \quad \frac{\mathrm{d} v}{\mathrm{~d} t}=F(t, x)
$$

this method becomes

$$
\begin{gathered}
x_{n+1}=x_{n}+h v_{n}, \quad v_{n+1}=v_{n}+h F\left(t_{n}, x_{n}\right), \quad h=\Delta t, \\
x=a+\omega ; y=b+c \omega ; p=c+\mathrm{F} \omega
\end{gathered}
$$

(Euler E366, Liber I, Pars II, Sectio I, Caput XII, Problema 137, §1082); Euler's $x, y, p, \omega, a, b, c$ are our $t, x, v, h, t_{0}, x_{0}, v_{0}$, respectively. The modus operandi of this method is illustrated in Figure 1.2: the solution point moves from the initial point $x_{0}$ with constant initial velocity $v_{0}$ during the time interval $h$ to $x_{1}$ (first formula of (1.4)). The initial velocity is updated by using the force $F\left(t_{0}, x_{0}\right)$ evaluated at the initial point (second formula of (1.4)) to obtain $v_{1}$, which is then used to move the solution point during the second time interval to $x_{2}$, and so on.

Euler did not, perhaps, require much genius to design this primitivelooking method, but he needed great genius to understand that it works, despite all these accumulated truncation errors and their propagation. He demonstrated the convergence of the solution, as $h \rightarrow 0$, for several examples. We demonstrate the convergence of the solution for problem (1.1) graphically in Figure 1.3. A formal convergence proof, using what was later called a Lipschitz condition, was given by Cauchy in 1824 .

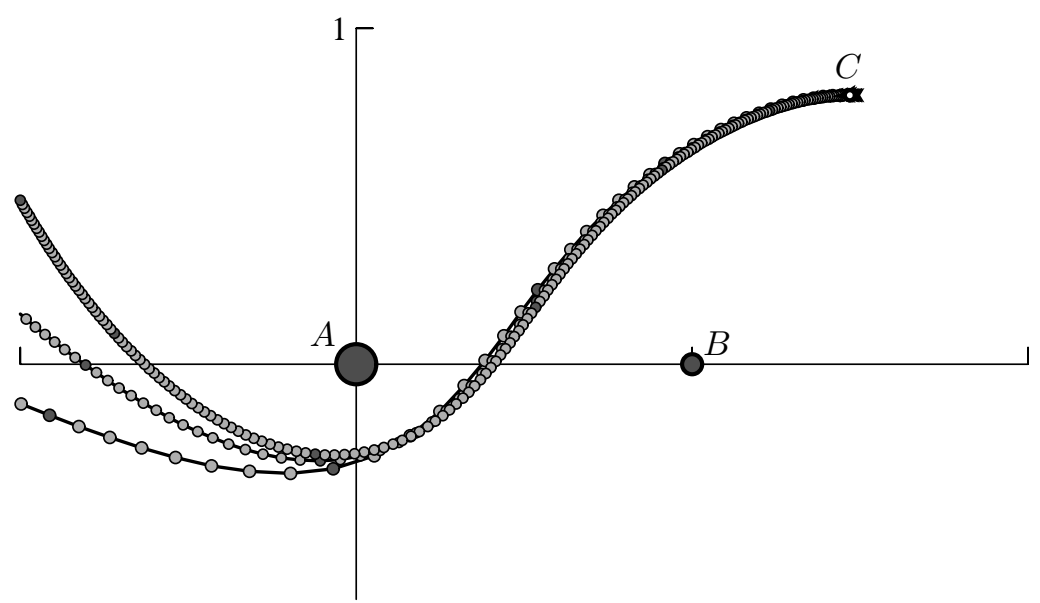

Figure 1.3. The solution of the problem with two fixed mass points using Euler's method for $h=1 / 32, h=1 / 64$ and $h=1 / 128$. 


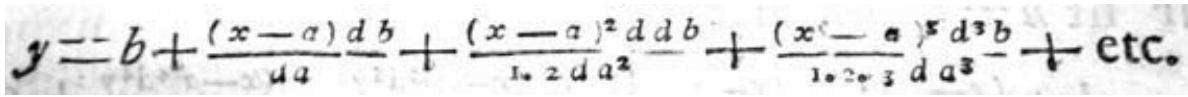

$$
\begin{aligned}
& \frac{d d y}{d x^{2}}=\left(\frac{d v}{d x}\right)+V\left(\frac{d v}{d y}\right) \\
& \frac{d^{3} y}{d x^{3}}=\left(\begin{array}{l}
d d v \\
d x^{2}
\end{array}\right)+\left(\frac{d V}{d x}\right)\left(\frac{d V}{d y}\right)+2 V\left(\frac{d d V}{d x d y}\right)+V\left(\frac{d V}{d y}\right)^{2}+V V\left(\frac{d d V}{d y^{2}}\right) .
\end{aligned}
$$

Figure 1.4. Euler's third-order Taylor method.

\subsection{Euler's higher-order methods and implicit methods}

A couple of paragraphs later (in $\S 656$ of E342), Euler realizes that one can very much improve ('magis perficere') the above method ('methodum praecedentem'), by adding, at each step, to the term $y_{n+1}=y_{n}+h V\left(x_{n}, y_{n}\right)$ higher-order terms of the Taylor series

$$
y_{n+1}=y_{n}+h y_{n}^{\prime}+\frac{h^{2}}{1 \cdot 2} y_{n}^{\prime \prime}+\frac{h^{3}}{1 \cdot 2 \cdot 3} y_{n}^{\prime \prime \prime}+\cdots,
$$

computing the higher derivatives of $y^{\prime}=V(x, y)$ by implicit differentiation $y^{\prime \prime}=\frac{\partial V}{\partial x}+\frac{\partial V}{\partial y} \cdot y^{\prime}$, etc. (see Figure 1.4).

It can be observed graphically in Figure 1.5 that this same method, applied with order 3 (up to this order, Euler wrote the derivatives explicitly) to our problem with two fixed mass points, really improves the solution, especially if the step-size $h$ becomes small.

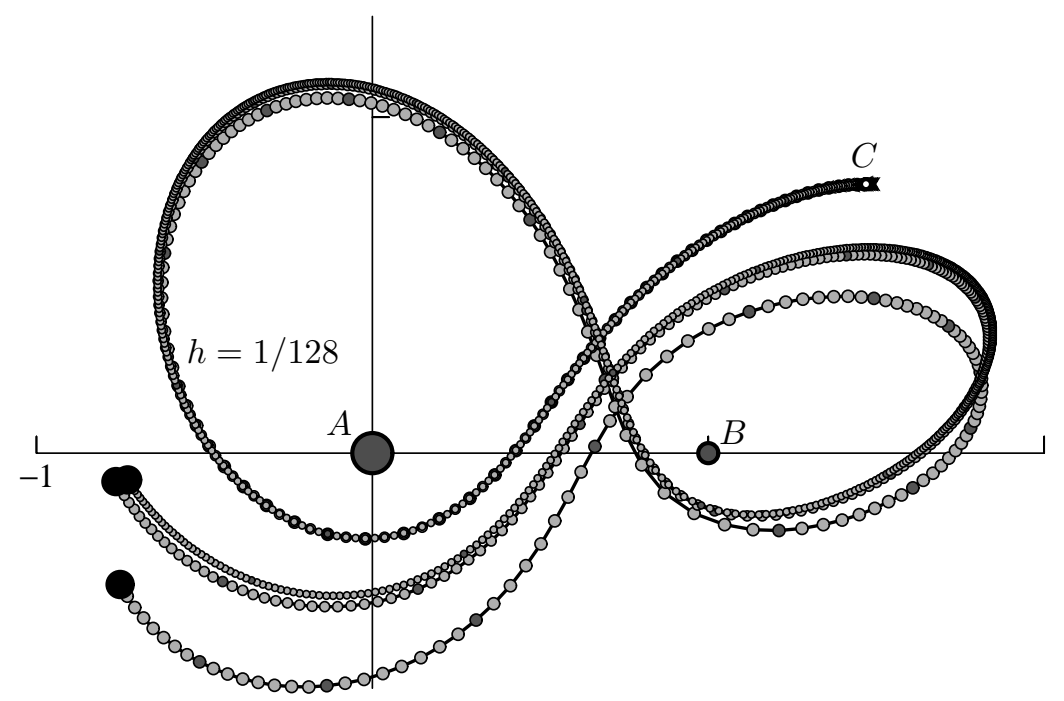

Figure 1.5. The solutions using Euler's third-order method for $h=1 / 32, h=1 / 64$ and $h=1 / 128$. 


\section{$y=b+\frac{(x-2) d y}{d x}-\frac{(x-a)^{2} d d y}{1+2 d x^{2}}+\frac{(x-a)^{3} d^{3} y}{1 \cdot 2 \cdot 3^{3} x^{5}}-\frac{(x-a)^{4} d^{4} y}{1+2 \cdot 3 \cdot 4 d x^{4}}+$ etc.}

Figure 1.6. Euler's third-order implicit 'Obreschkoff' method.

But what represents the formula reproduced in Figure 1.6, with its curious sign changes, which Euler had written half a page earlier? Well, we can observe that the initial value $b$ and the solution value $y$ have been interchanged. This is the implicit Euler method for order 1 and represents the so-called Hermite-Obreschkoff methods of the first column of the Padé table for higher orders; see Hairer, Nørsett and Wanner (1993, Section II.13). Euler realizes that the application of such a formula requires the solution of an 'aequatio algebraica'.

\subsection{Recursive computation of Taylor coefficients}

At the beginning of the era of automatic electronic calculating machines, many authors had discovered independently the possibility of computing, in a more or less general setting, the Taylor coefficients of the solutions of differential equations in a differentiation-free recursive algorithm. ${ }^{1}$ We demonstrate the method using the example $y^{\prime}=x^{2}+y^{2}$. Set

$$
y\left(x_{0}+h\right)=y_{0}+h y_{1}+h^{2} y_{2}+h^{3} y_{3}+h^{4} y_{4}+\cdots, x^{2}=x_{0}^{2}+2 x_{0} h+h^{2},
$$

develop

$$
\begin{aligned}
& y^{\prime}=y_{1}+2 y_{2} h+3 y_{3} h^{2}+4 y_{4} h^{3}+\cdots \\
& =x_{0}^{2}+2 x_{0} h+h^{2} \\
& +y_{0}^{2}+2 y_{1} y_{0} h+2 y_{0} y_{2} h^{2}+2 y_{0} y_{3} h^{3} \\
& +y_{1}^{2} h^{2}+2 y_{1} y_{2} h^{3}+\cdots
\end{aligned}
$$

and compare coefficients. In each column $y_{n+1}$, say, appears above terms of lower index, which allows us to compute them recursively via

$y_{1}=x_{0}^{2}+y_{0}^{2}, 2 y_{2}=2 x_{0}+2 y_{1} y_{0}, 3 y_{3}=1+2 y_{0} y_{2}+y_{1}^{2}, 4 y_{4}=2 y_{0} y_{3}+2 y_{1} y_{2}$,

and so on. This can be turned into the following general algorithm.

Suppose that $f$ of $y^{\prime}=f(x, y)$ is composed of elementary functions, such as $r=p q$ or $r=p^{c}$ with $c$ a constant. Then each of these functions is replaced by a formula computing the $i$ th term of the Taylor coefficient of $r$

1 This author, in his very first book, written in 1968, compiled the following list: J. F. Steffensen 1956, A. Gibbons 1960, W. Gautschi 1966, R. E. Moore 1966, I. Mennig 1964, Miller-Hurst 1958, E. Rabe 1961, E. Fehlberg 1964, Deprit-Zahar 1966, Leavitt 1966, Richtmyer 1957, Shanks 1964, Chiarella-Reichel 1968, A. J. Strecok 1968. 


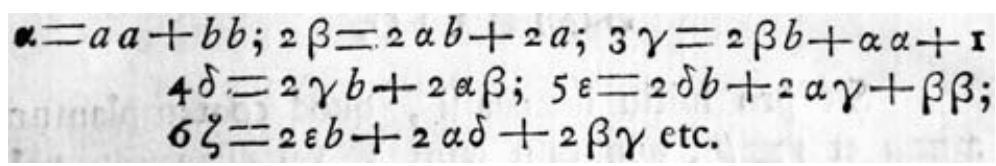

Figure 1.7. Euler's recursive differentiation-free computation of the Taylor coefficients of the solutions of $y^{\prime}=x^{2}+y^{2}$.

from coefficients of lower order of $r$, or of the same order of $p$ and $q$ :

$$
\begin{gathered}
r=p q: \Rightarrow r_{i}=\sum_{j=0}^{i} p_{j} q_{i-j}, \quad i=0,1,2, \ldots \\
r=p^{c}: \Rightarrow r_{0}=p_{0}^{c}, r_{i}=\frac{1}{i p_{0}}\left(\sum_{j=0}^{i-1}(c i-(c+1) j) r_{j} p_{i-j}\right), \quad i=1,2, \ldots
\end{gathered}
$$

Thus the following scheme computes all derivatives recursively:

$$
\begin{aligned}
& \left.\begin{array}{ccccc}
x_{0}, y_{0} & \mapsto \cdots & p_{0}, q_{0} \rightarrow r_{0} & \cdots \mapsto & f_{0} \\
\cdots & \cdots & & \cdots
\end{array}\right) \\
& \left(\begin{array}{ccccc}
y_{1}=f_{0} & \mapsto \cdots & p_{1}, q_{1} \rightarrow r_{1} & \cdots \mapsto & f_{1} \\
\cdots & \cdots & & \cdots
\end{array}\right) \\
& \oint y_{2}=\frac{1}{2} f_{1} \mapsto \cdots \quad p_{2}, q_{2} \rightarrow r_{2} \quad \cdots \mapsto \quad f_{2} \\
& \text { ( } \begin{array}{ccc}
\cdots & \cdots & \cdots
\end{array}
\end{aligned}
$$

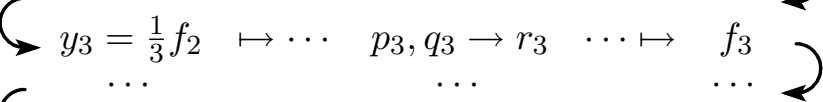

$$
\begin{aligned}
& \zeta y_{4}=\frac{1}{4} f_{3} \quad \text { etc., }
\end{aligned}
$$

to any order. And what is the surprise? If we compare Figure 1.7, which is copied from $\S 663$ of E342, with the formulas of (1.6), we see that Euler invented this too. ('Uti haec methodus simplicior ...')

\subsection{The 'symplectic' Euler method}

There is another way of improving Euler's method, not with the heavy machinery of higher derivatives, but with a small and clever modification of the formulas. We saw in Figure 1.2 that with Euler's method (1.4) the velocity updates $v_{1}-v_{0}$ are computed from the force $F$ at the point $x_{0}$, which lies just outside the interval of action of these two velocities. Hence we obtain a much more symmetric situation if we update the velocity with the force evaluated at the point $x_{1}$, and have in general

$$
x_{n+1}=x_{n}+h v_{n}, \quad v_{n+1}=v_{n}+h F\left(t_{n+1}, x_{n+1}\right), \quad h=\Delta t .
$$

This modification is free of charge: it just requires us to exchange two lines of the code, but leads to a significant increase of the performance, as can be seen in Figure 1.8. 


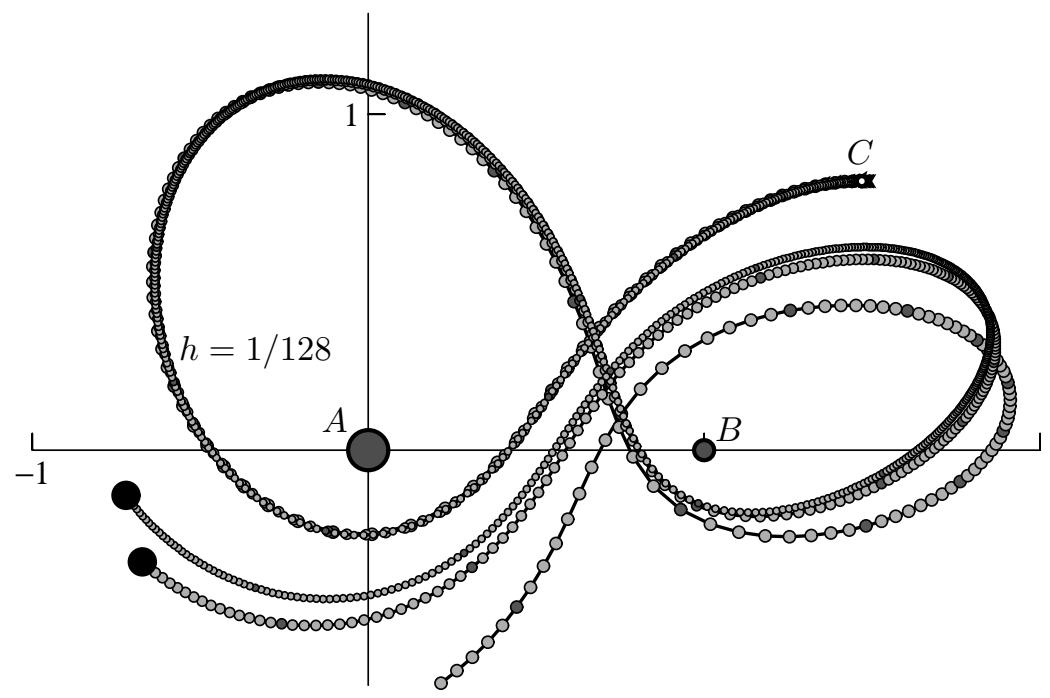

Figure 1.8. The solutions with the symplectic Euler method for $h=1 / 32, h=1 / 64$ and $h=1 / 128$.

This method, for reasons which are explained in Hairer, Lubich and Wanner (2006, Section VI.3), bears the name symplectic Euler method, and was designed by De Vogelaere (1956) for computations in nuclear physics. This very first paper on symplectic integration was so ahead of current research interests that it never found a publisher.

Now, after all we have seen above, we might ask if there is yet another miracle, and whether this method, too, can be found somewhere in Euler's work? No, this miracle has not taken place, but another miracle has: this method appears in Newton's Principia Mathematica, from 1687, and will play an important role in the discussions below. Did you say 1687, the reader may ask, a century earlier than Euler? Yes, but it was then used inversely, as we will see in the following sections.

\section{2. 'Inverse' numerical methods}

How are the differential equations for modelling phenomena of nature justified? We will see the surprising fact that numerical methods for ODEs are older than ODEs themselves, but were used inversely to establish these ODEs. Here are some examples.

\subsection{The tractrix of Leibniz}

During the years 1674/75 young Leibniz was visiting Paris and received from Christiaan Huygens his first introduction to 'modern' mathematics. During this visit, the famous architect and medical doctor Claude Perrault 
stated the following challenge: Which curve is described by a silver pocket watch ('horologio portabili suae thecae argentae'), when it is pulled across the table, where the end of the chain moves on a straight line (see (2.1) left)? Today's students are told that 'trivially' this curve must have a tangent of constant length $a$ and that therefore we have to solve

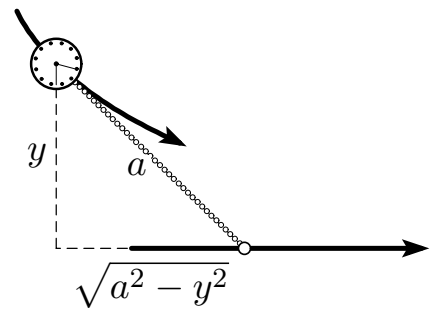

$$
\frac{\mathrm{d} y}{\mathrm{~d} x}=-\frac{y}{\sqrt{a^{2}-y^{2}}}
$$

which can be solved by methods revealed by Euler (E342 1768), namely, separation of the variables and then an integral containing a square root of a quadratic polynomial.
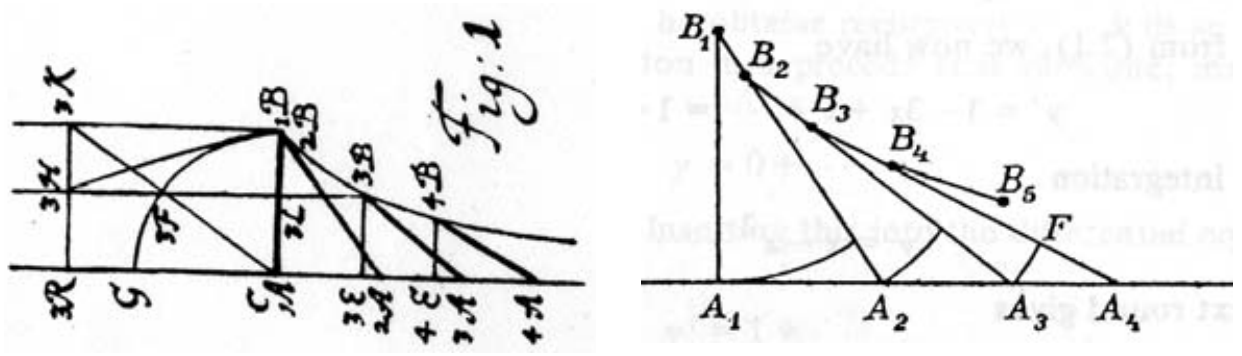

Figure 2.1. Drawings by Leibniz (left) and Kowalewski (right).

But for Leibniz who, as one of the inventors of differential calculus, was certainly not a stupid man, this conclusion was not so easy (see Figure 2.1, from Leibniz (1693); the drawing by Kowalewski, in his translation of 1908, is even clearer): physical intuition tells us that pulling the watch with a finger along a line is the same as rotating the chain, which is assumed to be without weight, by a small angle to the right, then pulling in the direction of the chain, until the imposed line is reached again, and so on. The polygon $B_{1}, B_{2}, B_{3} \ldots$, which we obtain in this way, is precisely the result of the implicit Euler method applied to equation (2.1). We see that this differential equation is justified through the inverse use of the implicit Euler method.

A brilliant history of the tractrix, including a detailed description from the earliest publications up to later developments in the 20th century, has recently been published by Tournès (2009). In particular, it contains on page 11 an autograph drawing by Jacob Bernoulli (dated before 1692) explaining the properties of the curve using the explicit Euler method. 

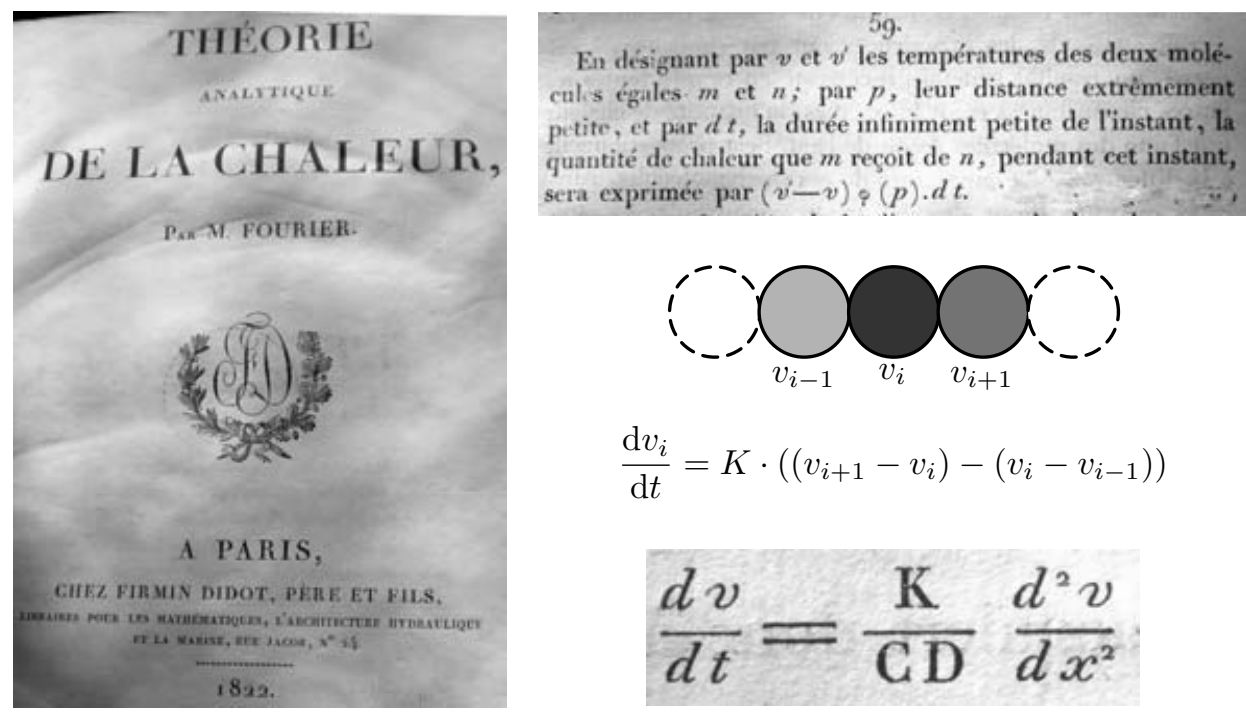

Figure 2.2. The discovery of the heat equation by Fourier (1822).

\subsection{Fourier's heat equation}

One of the most important books of science in the nineteenth century, La Théorie Analytique de la Chaleur by Fourier (1822), originates from a manuscript sent to the French Academy of Sciences in 1807, which was rejected due to 'lack of rigour' and could only be published after Lagrange's death. It is here that Fourier discovers the parabolic partial differential equation which governs the transport of heat, and whose solution led to the important concepts of separation of variables, eigenvalue problems, Fourier series and Fourier transform.

We see in Figure 2.2 the first publication of this equation, and in words (not formulas) Fourier's motivation for justifying it: in $\S 59$ he applies Newton's observation that the quantity of heat passing from one soup pot to another is proportional to the difference of the temperatures, on the molecular level. Therefore, if $v_{i}$ is the temperature of the $i$ th molecule (Fourier used the symbols $v$ and $v^{\prime}$ ), the heat it receives from the right-hand neighbour during an instance 'infiniment petite' of time is $\mathrm{d} v_{i}=K \cdot\left(v_{i+1}-v_{i}\right) \cdot \mathrm{d} t$; similarly the heat received from the left-hand neighbour will be $\mathrm{d} v_{i}=K \cdot\left(v_{i-1}-v_{i}\right) \cdot \mathrm{d} t$. The sum of these two terms is a second-order finite difference of the values $v_{i-1}, v_{i}, v_{i+1}$; when the distance of the molecules tends to zero, this expression will become the second partial derivative with respect to space. The heat equation is thus justified by the method of lines applied inversely. 

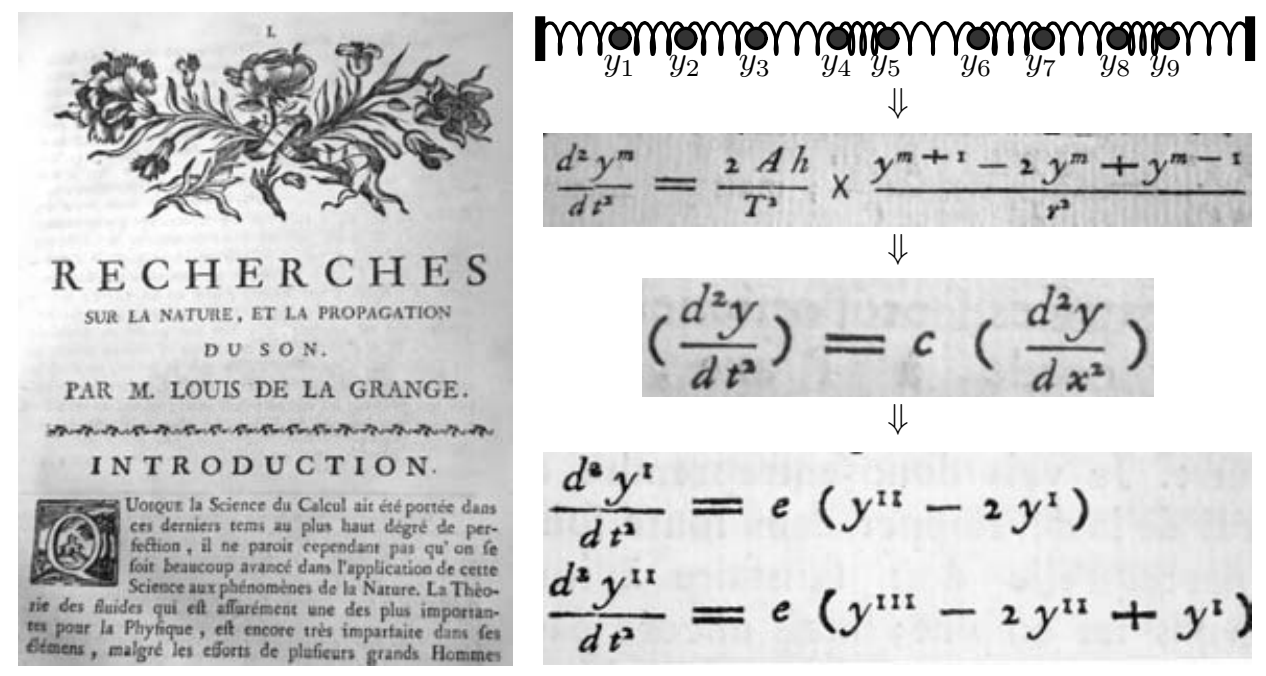

Figure 2.3. The discovery of the equation of sound by Lagrange (1759).

\subsection{Lagrange's theory of sound}

In Lagrange's paper of 1759, one of the first papers with which the young rising scientific star filled the newly founded Miscellanea Taurinensia, the above ideas are explained even more clearly: the air is thought to consist of a sequence of molecules, tied together with elastic forces. If we denote by $y_{i}$ the displacement of the $i$ th molecule, then the elastic forces acting from the left-hand and the right-hand neighbour are, following Hooke's law, proportional to the differences $y_{i-1}-y_{i}$ to the left, and $y_{i+1}-y_{i}$ to the right. The sum of these two forces is again a second finite difference and must be proportional to the acceleration $\frac{\mathrm{d}^{2} y_{i}}{\mathrm{~d} t^{2}}$. This gives the first formula in Figure 2.3 (right). The inverse use of the method of lines then leads to the partial differential equation for sound (second formula). In order to solve it, Lagrange proceeds back to the method of lines (third formula) which represents a linear system of ODEs with constant coefficients, which Lagrange solved using the 'now-so-familiar formulas' of Euler. After lengthy calculations, Lagrange finally stood at the door of Fourier series but did not open it; for more details see Hairer et al. (1993, pp. 28-29).

\subsection{Euler's equations for variational calculus}

We all learned in analysis lectures that Euler found his famous differential equation for a general variational problem,

$$
J=\int_{a}^{b} Z \mathrm{~d} x=\min ! \text { vel } \max !
$$



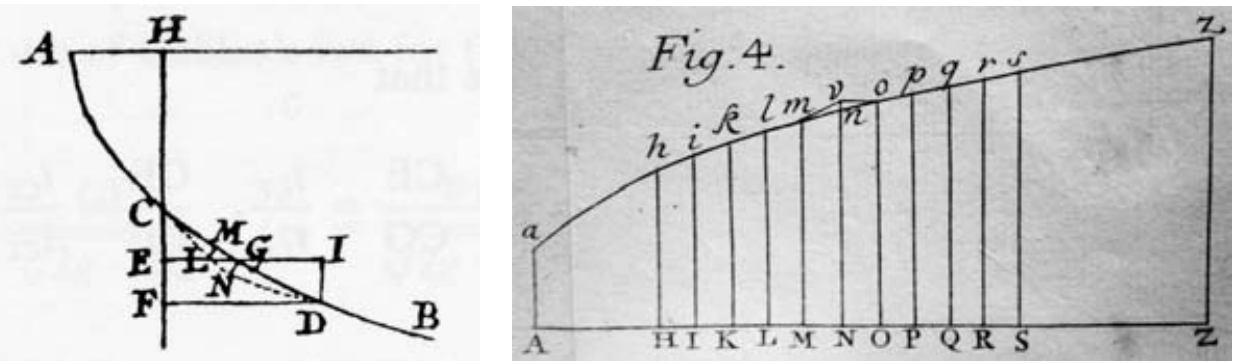

Figure 2.4. The 'variation' of one single $y$-value in Jacob Bernoulli's solution of the brachistochrone problem (left) and in Euler's derivation of his general formula (right).

where $Z=Z(x, y, p)$ is an arbitrarily given function of $x, y$ and $p=\frac{\mathrm{d} y}{\mathrm{~d} x}$, in his masterpiece, Euler E65 (1744), and that the proof usually given is due to Lagrange in 1755. This leads naturally to the question: How did Euler himself discover this equation, which enabled him to solve many dozens of such problems of all kinds? We will see that Euler's original route, displayed in Figure 2.4 (right), is quite elegant.

The solution $y(x)$ is represented by a discrete sequence $y, y^{\prime}, y^{\prime \prime}, y^{\prime \prime \prime}, \ldots$ (in the figure $h, i, k, l, m, \ldots$; today we would say 'piecewise linear finite elements'), and the integral $J$ in (2.2) by a 'finite Riemann sum',

$$
J=Z d x+Z^{\prime} d x+Z^{\prime \prime} d x+Z^{\prime \prime} d x+\& c .
$$

This $J$ must be minimal for each choice of the values $h, i, k, l, m, \ldots$ We therefore differentiate it with respect to any of these (in Figure 2.4 Euler moves $n$ to $\nu$ ). We write (2.3) more explicitly, merging Euler's notation with ours, and replacing the derivative $p$ by a finite divided difference

$$
J=Z\left(x, y, \frac{y^{\prime}-y}{\mathrm{~d} x}\right) \mathrm{d} x+Z\left(x^{\prime}, y^{\prime}, \frac{y^{\prime \prime}-y^{\prime}}{\mathrm{d} x}\right) \mathrm{d} x+\cdots,
$$

differentiate with respect to $y^{\prime}$, which appears in three places, set this derivative to zero, and obtain

$$
\left(\boldsymbol{P}+\boldsymbol{N}^{\prime} d \boldsymbol{x}-\boldsymbol{P}^{\prime}\right)=0, \quad \text { where } N=\frac{\partial Z}{\partial y}, P=\frac{\partial Z}{\partial p} .
$$

This condition, which must hold everywhere, is precisely the differential equation

$$
N-\frac{d P}{d x}=\mathbf{0} \quad \text { or } \quad \frac{\partial Z}{\partial y}-\frac{\mathrm{d}}{\mathrm{d} x}\left(\frac{\partial Z}{\partial p}\right)=0,
$$

discretized by the implicit Euler method. Hence, again, the inverse use of this method, and all other numerical procedures applied before, establishes equation (2.6), the famous Euler's equation of variational calculus. 


\subsection{Conclusion}

We see from all the above examples that numerical methods are more than just simple recipes which allow lazy scientists to consign their duty to a computer, but they constitute, applied inversely, the foundations of the application of analysis to science. We conclude with a quotation due to Bertrand Russell:2

Although this may seem a paradox, all exact science is dominated by the idea of approximation.

B. Russell, The Scientific Outlook (1931)

\section{The origin of Kepler's laws}

Astronomy is older than physics. In fact, it got physics started by showing the beautiful simplicity of the motion of the stars and planets, the understanding of which was the beginning of physics.

R. Feynman (1963); published in Six Easy Pieces (1994), p. 59 [Gravity] was one of the first great laws to be discovered and it has an interesting history. You may say, 'Yes, but then it is old hat, I would like to hear something about a more modern science'. More recent perhaps, but not more modern. ... I do not feel at all bad about telling you about the Law of Gravitation because in describing its history and methods, the character of its discovery, its quality, I am being completely modern.

R. Feynman, Messenger Lectures (1964);

published in The Character of Physical Law (1967)

We now go back to the very beginning of science (see the first quotation from Feynman), or rather, to the beginning of modern science (second quotation, from a public lecture in which Feynman, soon to receive the Nobel Prize, spoke, not about recent discoveries in quantum electrodynamics, but, to the astonishment of everybody, about Kepler's and Newton's laws).

\subsection{Ptolemy $(\sim A D$ 150)}

The first great observer of the sky, with thousands of precise measurements of the position of the stars and planets, was Ptolemy, who lived around AD 150. His fundamental work, originally called the Great Collection, or,

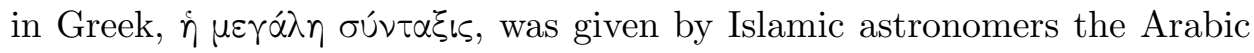
definite article 'al', to become the multilingual conglomerate Almagest. It was translated in the second half of the fifteenth century by Peurbach and

${ }^{2}$ Communicated to the author by Jan Lacki, Geneva. 

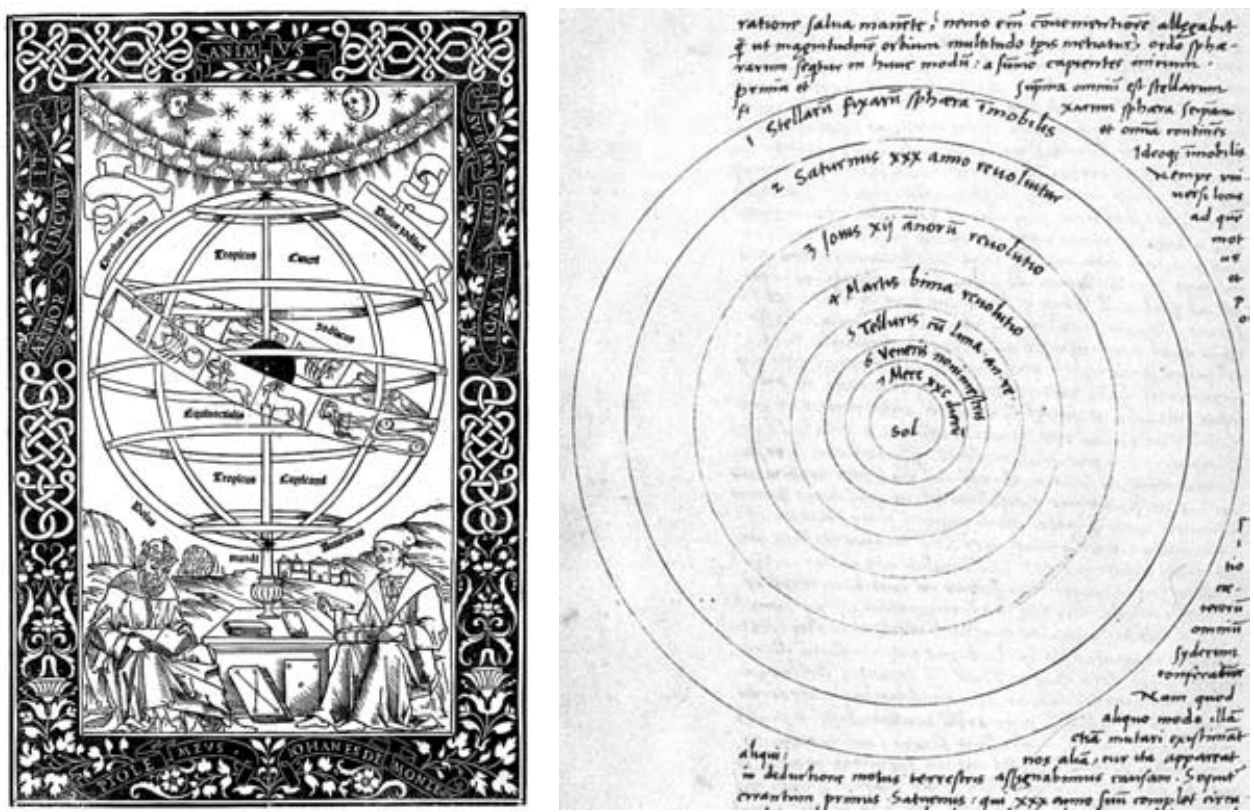

Figure 3.1. Left: Frontispiece of Regiomontanus' translation of Ptolemy's Almagest, published in 1496; we see the Earth in the centre, with the stars rotating around attached to a solid machinery. Right: A page of Copernicus' manuscript De Revolutionibus, representing the heliocentric system, published just before his death in 1543. Reproduced, with permission, from the copy of De Revolutionibus, call number BJ10000, in the Library of the Jagiellonian University, Kraków, Poland.

Regiomontanus and published in 1496 in Venice as one of the very first scientific books ever printed (see Figure 3.1, left). This book had a big influence on the astronomers of that time, in particular a young Polish fellow studying in Bologna, Nicolaus Copernicus. Ptolemy's conclusion after all his measurements is explained in the central drawing of Figure 3.2 (right): the Earth is in the centre, the Sun moves around the Earth, and the planets move on epicycles, circles whose centres move around the Earth.

\subsection{Nicolaus Copernicus (1473-1543)}

Kepler (1609, p. 131) wrote: 'I start by explaining these things in the Copernican setting, where they are easiest to understand.' Hence, the Copernican system is explained in the first image of Kepler's drawing in Figure 3.2 (right): the Sun is in the centre and all planets, including the Earth, rotate around the Sun on circles which are, however, in an eccentric position. This model, which makes no distinction between the Earth and the other planets, is the simplest of all, and therefore an attractive choice. An 

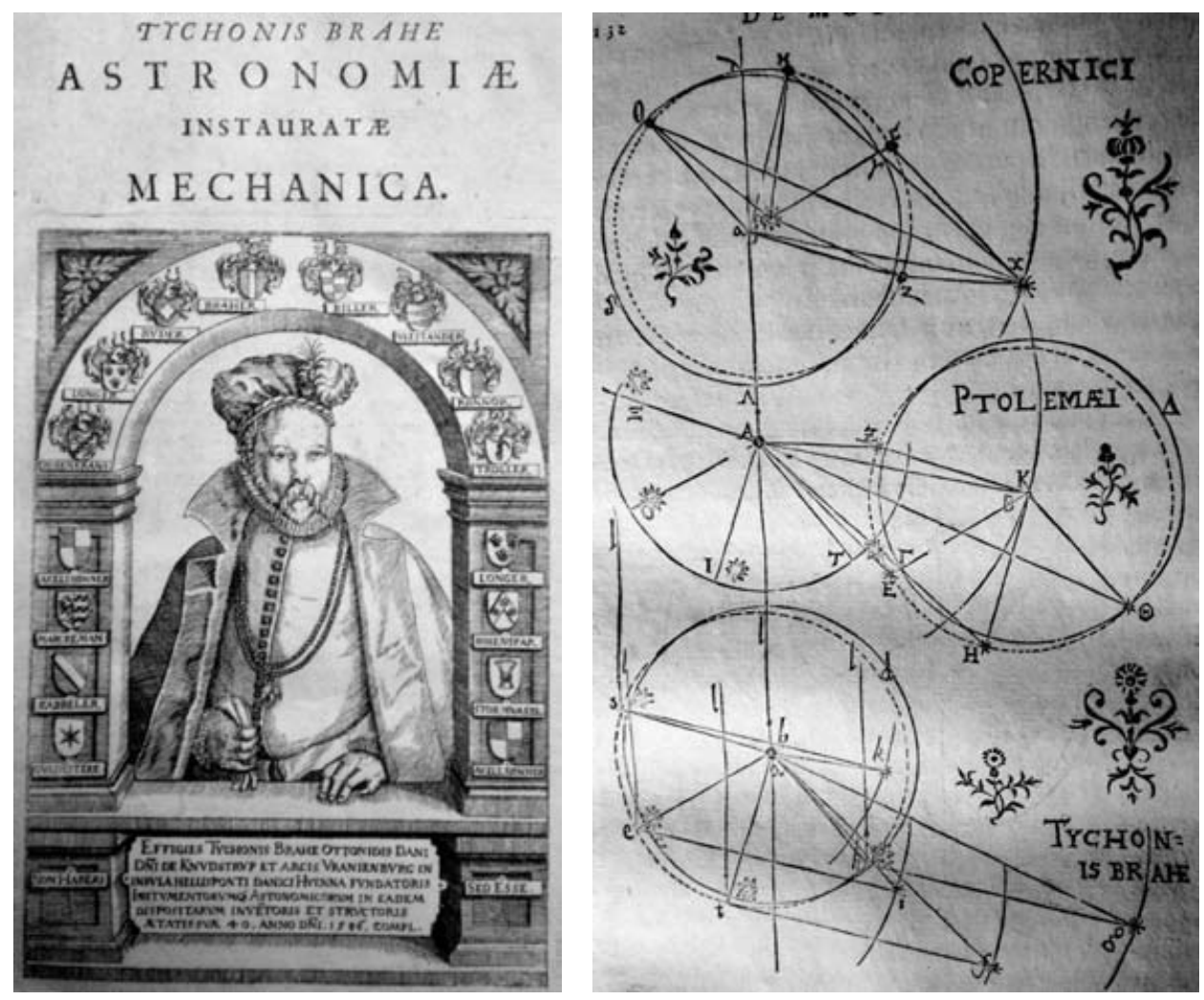

Figure 3.2. Left: Tycho Brahe (1546-1601). Right: Explanation of the three systems of Copernicus (above), Ptolemy (centre) and Tycho Brahe (below) in Kepler's Astronomia Nova (1609).

outermost circle ('sphaera immobilis') is then reserved for the fixed stars ('stellarii fixarii'; see Figure 3.1, right). Copernicus, who had obtained this result after life-long effort and observations, hesitated to make it public, but this was finally achieved with the help of the German mathematician G. J. Rheticus, just before Copernicus' death in 1543.

\subsection{Tycho Brahe (1546-1601)}

Tycho Brahe was a Danish nobleman who became impassioned by astronomy after a solar eclipse of 1560 , the supernova of 1572 and the comet of 1577. The imperfection of existing astronomical predictions, based on Ptolemy's calculations, motivated him to become 'a second Ptolemy', by building the huge astronomical observatory Uraniborg on the island of Hven, where, over many years, he made thousands of astronomical measurements with unequalled precision (accurate to 1 arc minute). After problems with the new king, he left Denmark in 1597 with all his tables and went to Prague. 
Tycho did not accept the Copernican system, because if the Earth were moving around the Sun, then the fixed stars must show a parallax, which, however, could not be observed. ${ }^{3}$ Therefore, Tycho's model of the universe was as follows (see the third drawing in Figure 3.2, right): the Sun rotates around the Earth on an eccentric circle, and the planets rotate around the Sun, also on eccentric circles. The sphere with the fixed stars does not move.

\subsection{The Ptolemy-Copernicus-Brahe model for planetary motion}

As long as we are only interested in the relative position of a planet with respect to the Sun, i.e., in the geometry of such an orbit, and not its physical quantities such as forces and accelerations, it does not matter which body is moving and which is not (since we are in Denmark, we may say 'to move or not to move, that is the question ...'). In this case, all three above models are equivalent and state the following (see Figure 3.3).

(1) The planets move around the Sun on circular orbits. The centre $B$ of this circle is called the Mean Sun and its distance from the Sun $S$ is governed by the eccentricity. This law describing the geometric form of the orbit is called in the old literature 'inequalitas prima'.

(2) The speed of the planet on this orbit, which has been observed being faster close to the Sun and slower away from it, is governed by a punctum aequans $C$, which is at the same distance from $B$ as $S$, in the opposite position. Seen from this punctum aequans, the planets move at constant angular speed. This law is called 'inequalitas secunda'.

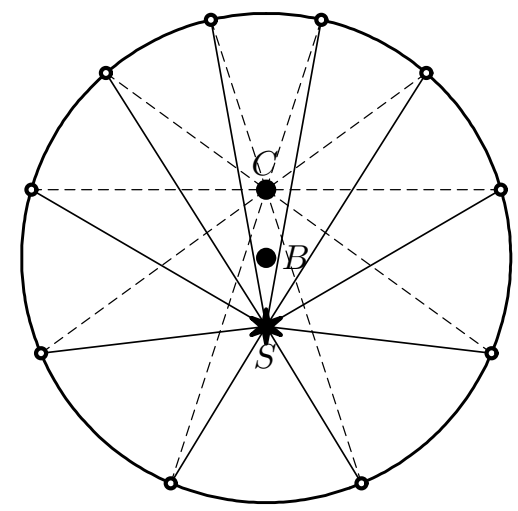

Figure 3.3. The Ptolemy-Copernicus-Brahe model for planetary motion: $S$ the Sun, $B$ the Mean Sun, $C$ the punctum aequans.

${ }^{3}$ Because of the tremendous distance of the fixed stars, their parallaxes, which were finally discovered more than 200 years later (by Bessel in 1838), required more than a hundred times greater precision $(<0.3$ arc seconds). 
In numerical calculations lasting years, Tycho tried to adjust the three parameters (radius, eccentricity and inclination of the line $C S$ ) of each of the planetary orbits for all his observations.

This worked fine for all planets except for Mars!

When young Johannes Kepler, who had fled Graz because of religious persecution, arrived in Prague, Tycho gave him the data of Mars to study. It is suspected that Tycho, who found the young man too self-assured and ambitious, wanted to cool him down with an apparently impossible task.

\subsection{Kepler's Astronomia Nova}

During the year 1605 Kepler finally unveiled the secret of the orbit of Mars, four years after the sudden death of Tycho. However, owing to differences with Tycho's family, lack of money and the tremendous size of Kepler's manuscript, the book could not be published until 1609. In the same year, Galileo pointed his first telescope towards the sky. Both events, 400 years ago, can be said to mark the birth of modern astronomy, and thus of all modern science.
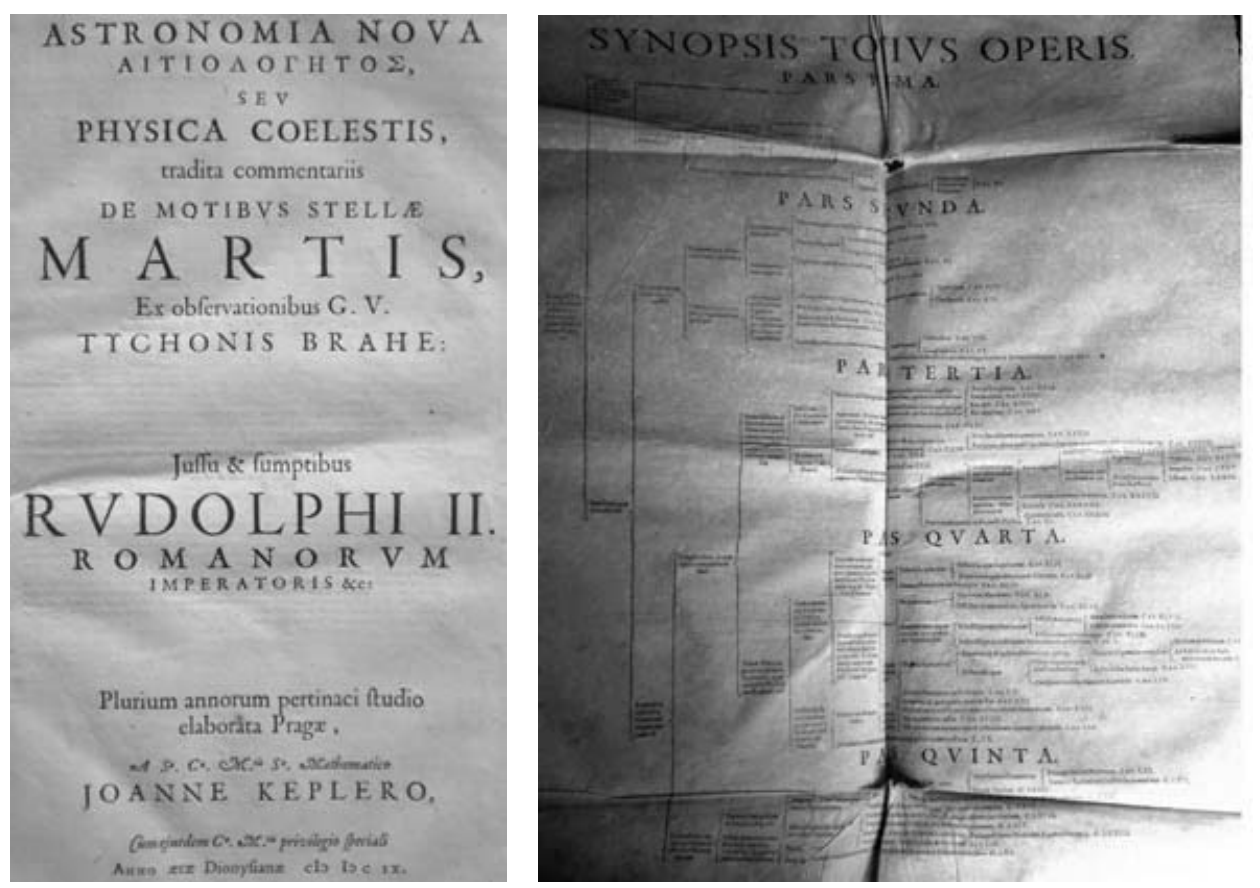

Figure 3.4. Frontispiece and synopsis of Kepler's Astronomia Nova. 
We see in Figure 3.4 (left) the frontispiece of this work. Following the title, Kepler writes 'Aitı入orñós seu Physica Coelestis', which expresses that Kepler is also interested in the physical reasons for the movement of the planets, and not only in their geometry. Then comes 'of the movement of the star Mars from the observations of Tycho Brahe'. We see that Tycho's name is printed in precisely the same size as 'the mathematician Johannes Kepler' below. We also read 'after many years of pertinacious study in Prague'. The name of Emperor Rudolf II, who paid for all this, is in the same huge letters as the planet Mars.

After a long hymn of praise for the emperor, several poems and an obligatory page by Tycho's son-in-law F. G. Tengnagel praising Tycho, the book starts with an introduction, which sounds very 'modern', about the difficulty of writing (and reading) a mathematical book (see Figure 3.5).

The book, with its 340 quarto pages, arranged in 70 chapters, is indeed very difficult to read. To help the reader, Kepler included a 'Synopsis Totius Operis' on a huge sheet, which must be (carefully) unfolded from the book, listing all the 70 chapters in the form of a binary tree (see Figure 3.4, right). We see that the book consists of five parts; parts three (discovery of Kepler's Second Law) and four (Kepler's First Law) are the central core.

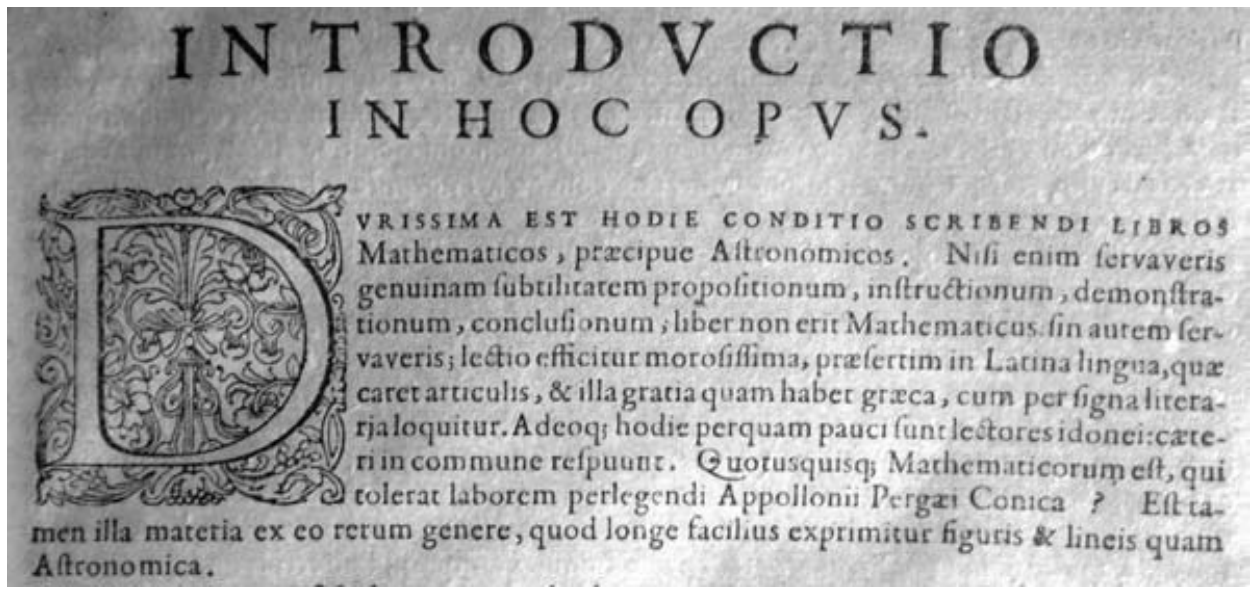

Figure 3.5. The 'very modern' introduction of Kepler's Astronomia Nova: 'Today the requirements for writing mathematical books, especially astronomical ones, are very hard. If you do not preserve the original details of the propositions, instructions, demonstrations and conclusions, the book will not be mathematical. If, however, you do preserve them, the book will be very boring to read.' 


\subsection{Kepler's Pars Secunda: 'Ad imitationem veterum'}

If you find these calculations tedious ('pertaesum'), then have pity on me ('jure mei te miserat'): I did them at least 70 times, losing a lot of time ('ad minimum septuagies ivi cum plurima temporis jactura').

Kepler, Astronomia Nova (1609), p. 95

In part two, Kepler tries to obtain the best possible results 'by imitating the Ancients' (the 'Ancients' are Ptolemy, Copernicus and Tycho Brahe, 25 years older than him). His main observation is that there is no convincing reason for the assumption that, in Figure 3.3, the punctum aequans $C$ is at the same distance from $B$ as the Sun $S$. Therefore he allows an arbitrary position for $C$, which increases the number of free parameters to 4 , hence these constants must be determined from 4 observations. This leads to very tedious calculations (see quotation), which Kepler solved by an iterative scheme similar to the regula falsi (see Figure 3.6). As a result, Kepler obtained a very precise model, the Hypothesis Vicaria, for the orbit of the Earth. The importance of this is the fact that only through knowing the distance of the Earth at any moment can the distances of the other planets be computed accurately, by measuring all the angles and using the sine theorem of trigonometry.
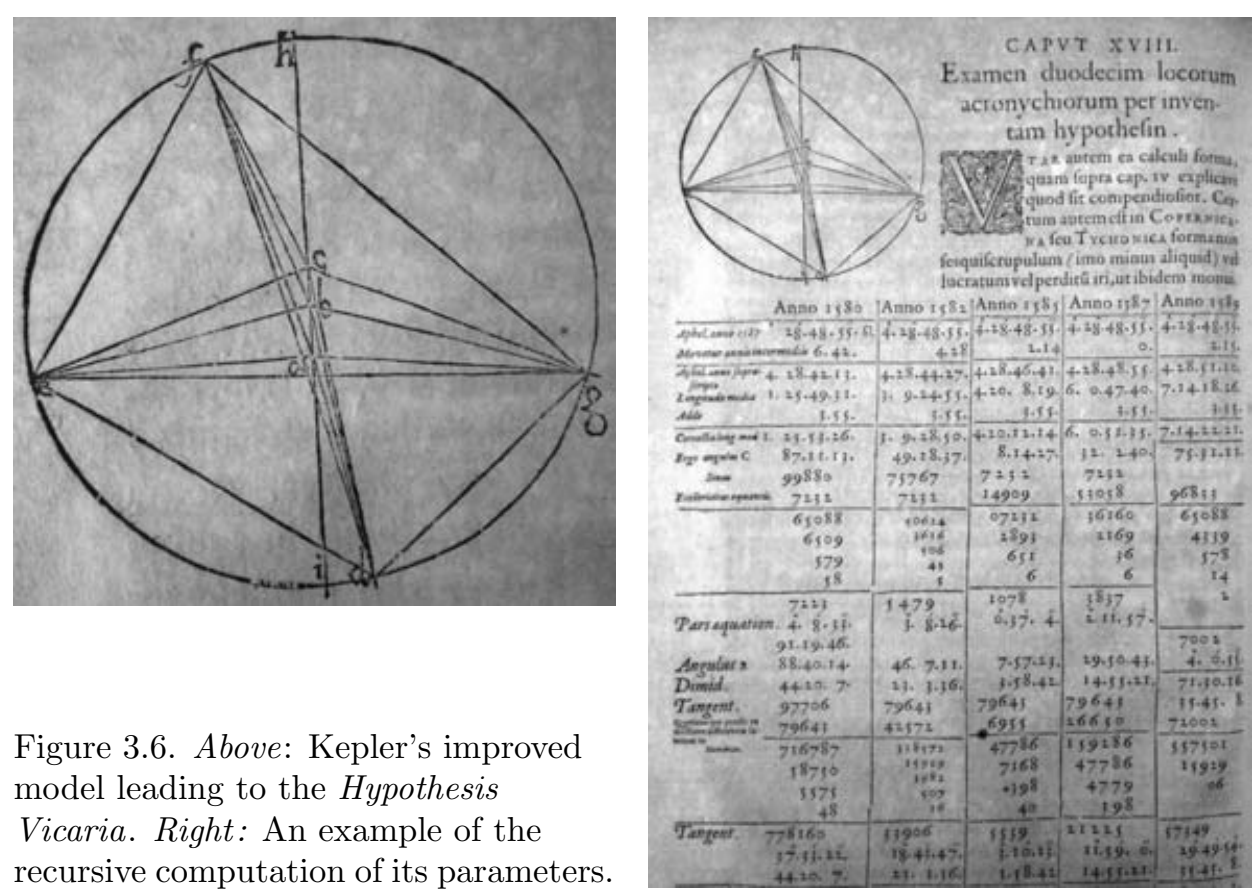

Figure 3.6. Above: Kepler's improved model leading to the Hypothesis Vicaria. Right: An example of the recursive computation of its parameters. 


\subsection{Kepler's Pars Tertia: 'Ex propria sententia' (his own opinion).}

In his third part (Chapters 22-40), Kepler wants to rid himself more and more of all this geometric thinking, with the punctum aequans $C$ as a kind of machine pushing the planets, and wants to accept only truly physical reasons, as announced in his title:

\section{A I T IO $\triangle$ O ГH TO $\mathrm{P}$ PHYSICA COELESTIS,}

These discussions fill Chapters 32-39. But if we throw away the punctum aequans, we have to replace it by something else. What could that be? The only really fixed object is the Sun, in the centre of the Universe ('I myself am of the opinion of Copernicus: I admit that the Earth is one of the planets', p. 170). After long deliberations about all possible reasons, forces, magnetic forces, the light coming from the Sun, wind from an ether, he finally concludes that planets must have a soul, which looks to the Sun and 'wishes' to move, seeing its diameter inversely proportional to the distance ('Is it so, Kepler, that you attribute two eyes to each planet? Not at all.' p. 191). 'You see thus, reader, with reflection and spirit' (p. 191), that the speed of the planets is inversely proportional to the distance (see Figure 3.7, left).

However, the arc length of a curve is a nasty mathematical expression: it involves Pythagoras' theorem and an uncomfortable square root. So, finally, inspired by the ideas of a great mathematical god, Archimedes,
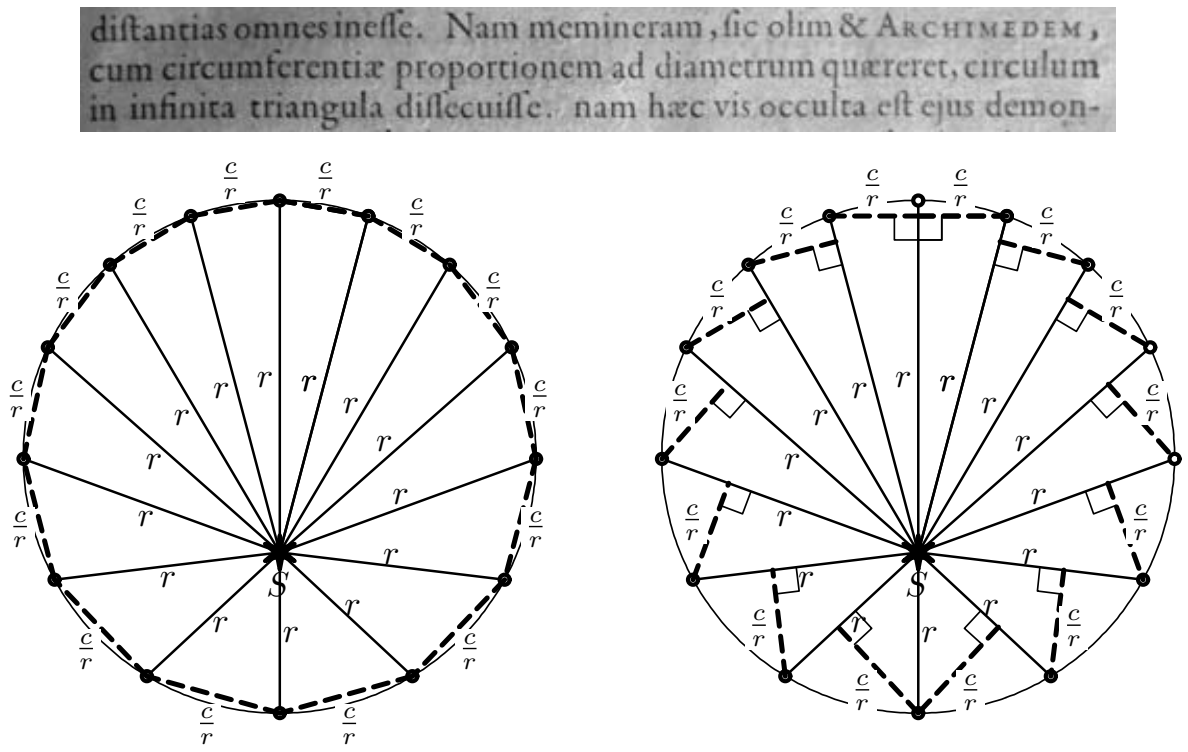

Figure 3.7. Left: Kepler's model from Chapter 39 with the speed inversely proportional to the distance. Right: Kepler's 'methodus imperfecta' from Chapter 40: equal times correspond to equal areas. 
he decides to replace the hypothenuse by the leg (the orthogonal distance of the rays) and arrive at the 'methodus imperfecta', saying that the heights of the triangles are inversely proportional to the distance, i.e., that the areas of the triangles for equal times are all the same (see Figure 3.7, right). In this way, Kepler's Second Law was finally discovered, and corresponded perfectly with the observations of Tycho.

\subsection{Kepler's Pars Quarta: the elliptic orbits}

The most difficult part was yet to come: the definite renunciation of all Greek and medieval thinking on circular orbits. This nearly hopeless struggle fills Chapters 41-55, until the epiphany in Chapter 56 (p. 267):

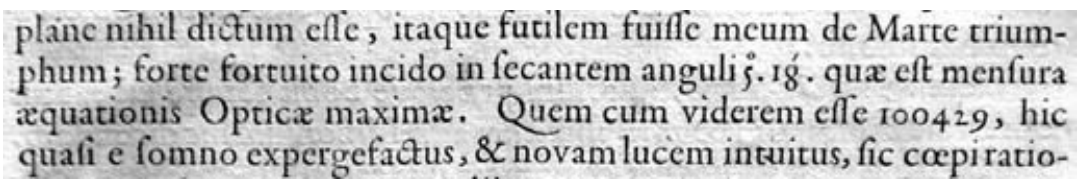

As I reflected ... that my triumph over Mars had been futile, I fell by chance on the observation that the secant of the angle $5^{\circ} 18^{\prime}$ is 1.00429 , which was the error of the measure of the maximal point.

I awoke as if from sleep, \& a new light broke on me.

This decisive discovery is explained in Figure 3.8 (right). If the orbit of Mars were a circle of radius 1 , then the eccentricity $e=O S$ is such that the angle $O B S$ would be $5^{\circ} 18^{\prime}$, where $B$ is the point with the largest elongation from the axis $S O C$. Therefore the distance $B S$ would be $1 / \cos 5^{\circ} 18^{\prime}=1.00429$. But Tycho measured 1 for this distance. Therefore, we should move the point $B$ to the point $B^{\prime}$, whose distance $B^{\prime} S$ is that of $B O$; in other words, we have to replace, once again, the hypothenuse $B S$ by the leg $B O$ ('Hence, what brought us to despair in Chapter 39, now changes here into an argument to attain the truth,' p. 267). Kepler applied the same recipe to other points: move the point $P$ to the position $P^{\prime}$ so that the length $P^{\prime} S$ is that of the leg $P R$, which is

$$
P^{\prime} S=P R=1+e \cos u,
$$

because the angle $u$, called the eccentric anomaly, reappears as angle $S O R$, so $O R=e \cos u$. Kepler finally concluded that 'these distances are confirmed by very numerous and very sure measurements' (Chapter 56, end).

In Chapters 58 and 59, Kepler finally achieves the proof that the orbit expressed by formula (3.1) represents an ellipse, with the Sun in one focus. The theory of conics had been developed to high perfection by Apollonius $(\sim 250 \mathrm{BC})$ and completed by Pappus ( AD 300-350), but then forgotten for more than 1000 years. One rediscovery, albeit in a very rudimentary form, had been by Kepler himself in 1604, so Kepler claimed his proof to be 

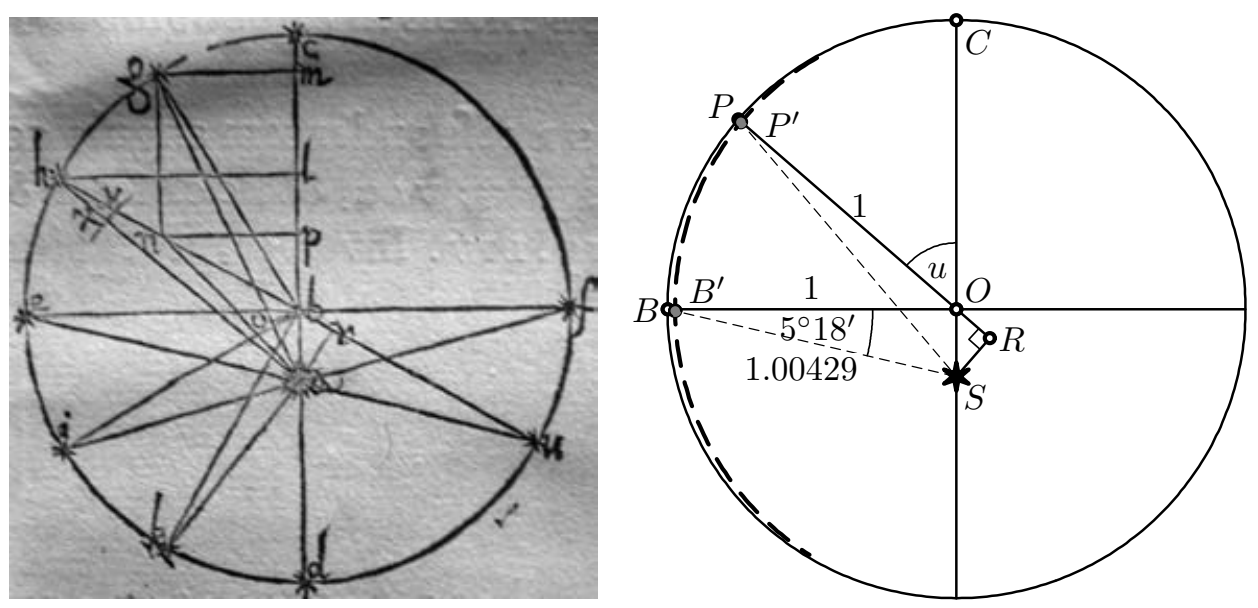

Figure 3.8. The discovery of Kepler's First Law (Chapter 56, p. 267): Kepler's drawing (left), modern drawing (right).

'although very sure, lacking art and not geometric' (p. 293). He concluded that the reader should consult the conics of Apollonius, 'which requires very strong meditations and reflections about these matters' (p. 295). In fact, formula (3.1) just expresses the result of Pappus that the distance $P S$ is in a constant ratio $e: 1$ with the distance of $P$ to a directrix.

In Chapter 60, finally, Kepler establishes for the area $P^{\prime} S C$, which by his Second Law is proportional to the elapsed time $t$, the expression

$$
\text { Const } \cdot t=\frac{B^{\prime} O}{2} \cdot(u+e \sin u)
$$

('composed of two portions of area, a sector and a triangle', p. 299). The solution of this equation allows one to find $u$ for any given time $t$, 'but I believe myself unable to solve it, and whoever shows the way would be for me a great Apollonius' (p. 300).

\subsection{Kepler's Third Law}

Work on the Astronomia Nova, with all its 'artless' numerical calculations and hazardous conclusions, was for Kepler something of an interruption to his true vocation, which was to unveil the harmonies of God's creations with the help of the beauty of mathematics, in particular geometry and music. ${ }^{4}$

4 'Geometria enim, ... Deo coaeterna, inque Mente divina relucens, exempla Deo suppeditavit, ... exornandi Mundi, ut is fieret Optimus et Pulcherrimus, denique Creatoris simillimus.' (p. 13; see also Gesammelte Werke, Vol. 6, pp. 104 and 489; 'Geometry, eternal as God, and shining out of the Divine Mind, has supplied God with the models for shaping the World, making it the Best and most Beautiful, hence similar to the Creator.') 


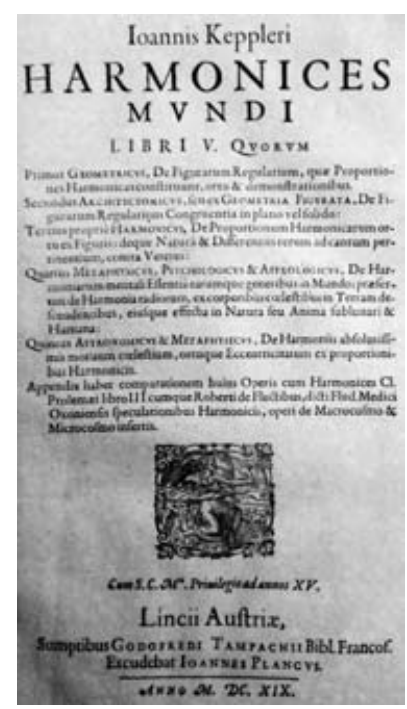

Researching harmonies ...

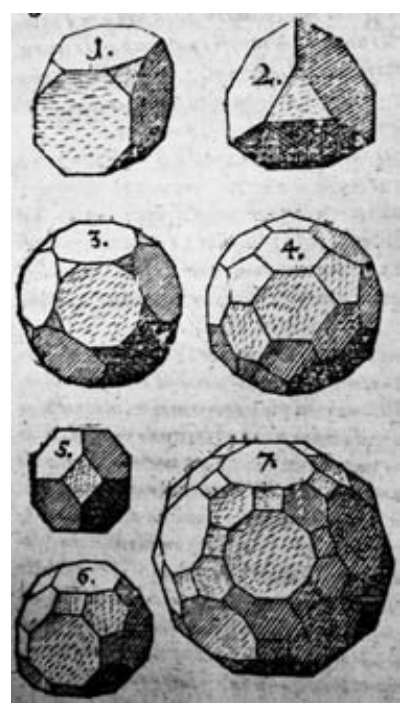

from geometry ...

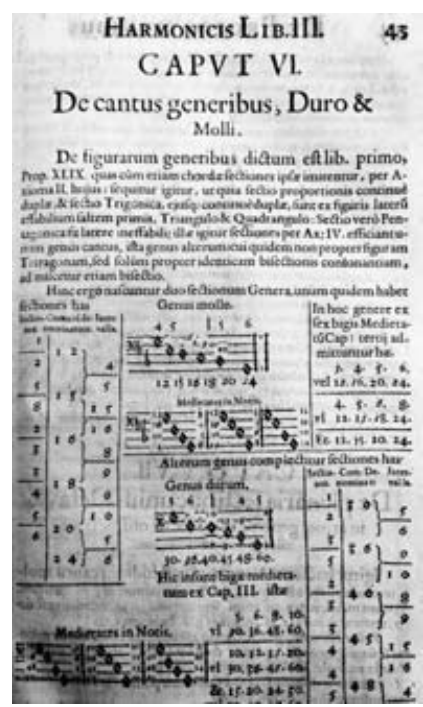

and music ...

Figure 3.9. Kepler's Harmonices Mundi (1619).

This research which he had begun in 1599 led finally to the Harmonices Mundi (Kepler 1619), in five books and an appendix (see Figure 3.9).

Although the books contain many beautiful results in mathematics, in particular the first rediscovery of the complete list of the 13 so-called Archimedean solids (some of which are shown in Figure 3.9, centre), the applications of all this to interesting results in astronomy led constantly to failure. Finally, the most famous result of this book was again discovered by vulgar numerical calculations and announced towards the end (Kepler 1619, Liber V, Caput 3, §8, p. 189) as follows:

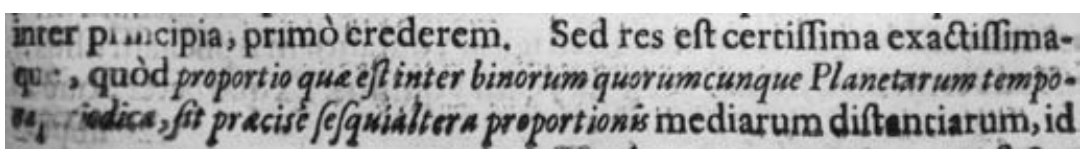

'It is extremely certain and extremely exact that the ratio of the time period for two planets is one and a half of the ratio of the mean distances.' In this way Kepler expresses the fact that

$$
T_{1} / T_{2}=\left(a_{1} / a_{2}\right)^{3 / 2}
$$




\subsection{Galileo Galilei (1564-1642)}

... io grandemente dubito che Aristotele non sperimentasse ...

(I have strong doubts if Aristotle did make any experiments ...)

Galileo's Discorsi (1638), First Day

De subiecto vetustissimo novissimam promovemus scientiam.

(We present an entirely new science on a very old subject.)

Galileo's Discorsi (1638), Third Day

Another important influence on modern science came from Galileo in Italy. A first manuscript by Galileo on mechanics, finished around 1629, was translated into French and published in 1634 by Marin Mersenne. He soon ran into problems with the Roman Curia, and his masterly publication of 1638, Discorsi e Dimostrazioni Matematiche, written in the form of discussions between three persons of different scientific level in six days, was smuggled out of Italy and published in the Netherlands.

In 1609, the same year in which Kepler's Astronomia Nova appeared, Galileo first directed his telescope towards the sky. His discovery of the satellites of Jupiter and the phases of Venus left no doubts about the Copernican system. At the same time, he tried to understand mechanics by experimenting with ropes, heavy stones and beams down on Earth (see Figure 3.10). While the first two 'days' of the Discorsi were written in popular Italian, for the third 'day' Galileo turned to serious Latin (see quotation), and laid the fundamental principles of an 'entirely new' mechanics, such as mass, forces and acceleration, as we know it today.
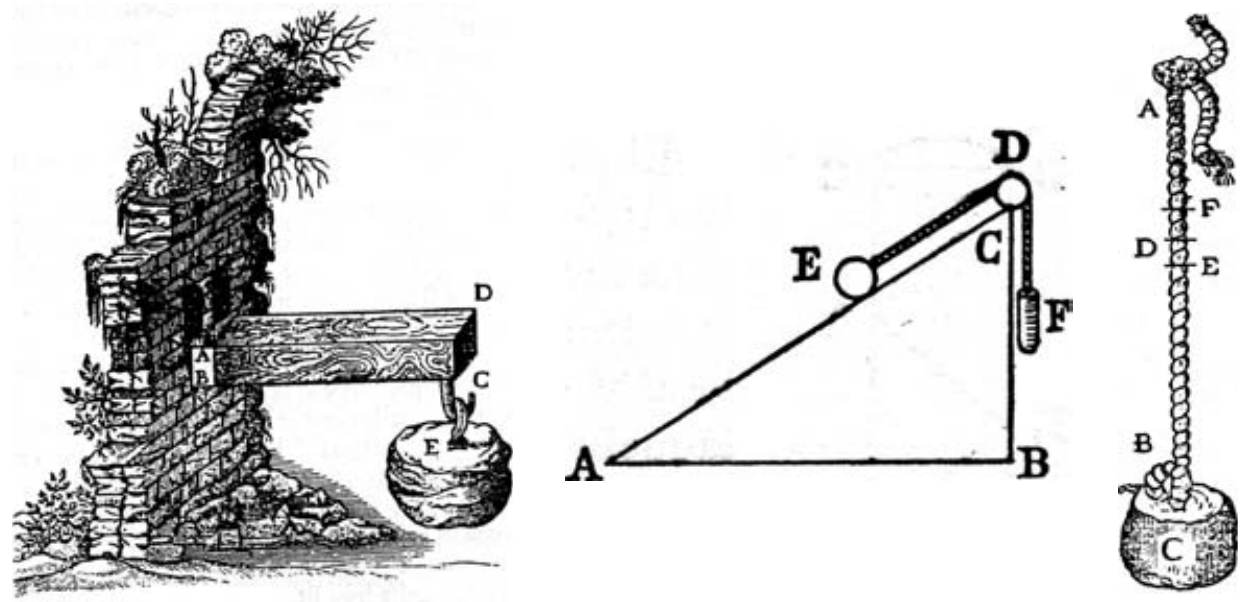

Figure 3.10. Illustrations from Galileo's Discorsi of 1638

(left and right), and his Mechanics of 1629 (centre). 


\subsection{Conclusion}

We conclude this section with a quotation from Kepler (1609, p. 95):

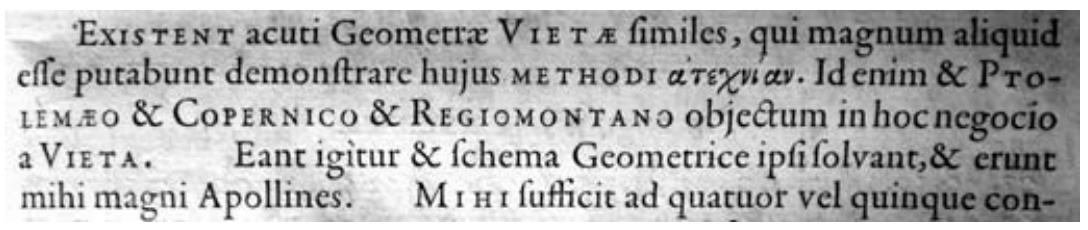

There might exist ingenious geometers, like Viète, who think they are doing something great in showing this [numerical] method to be artless. ${ }^{5}$ Indeed, Viète made this very criticism of Ptolemy, Copernicus \& Regiomontanus in his work. Let them step forward, then, \& solve the scheme geometrically. They will be great Apollos for me.

This great Apollo will lead us to the next section.

\section{Newton's discovery of the Law of Gravitation}

This sudden change of emphasis has been provoked by a visit from Edmund Halley (1656-1742), which probably took place in August [1684].

S. Mandelbrote, Footprints of the Lion (2001), p. 88

During the half-century which separated Kepler's works from Newton's studies in Cambridge, Kepler's laws slowly became known and accepted, not through the books we have cited above, but through the Rudolphine Tables (Kepler 1627), a huge compendium of more than 300 pages of tables for the positions of stars and planets, which Kepler computed with the help of his laws, and which became the universal tool for generations of astronomers - and astrologers. Also, Galileo's principles of mechanics became known, particularly in Cambridge through the lectures of Isaac Barrow, who had visited Paris and Florence in 1655/56.

It thus became a natural challenge to understand the principles governing the movement of the planets from a mechanical point of view. Newton, who was in priority dispute with Robert Hooke of London over this discovery, declared later that he had discovered all this during the plague years 1665/66, but no written evidence about this claim could be found in his manuscripts.

The first manuscript clearly showing Newton's ideas is one dating to 1684, initiated by a visit to Cambridge by Edmund Halley, bringing news from

${ }^{5}$ In fact, this is still a very 'modern' opinion. 
London (see quotation). All the illustrations produced below are reproduced from this manuscript. ${ }^{6}$ This manuscript (and others) later led to the epochmaking Principia (Newton 1687).

\subsection{Proof of Kepler's Second Law}

... what Newton writes is correct, clear, and short; in earlier works the brilliant diamonds of discovery lie concealed in an opaque matrix of wordy special cases, laborious details, metaphysics, confusion, and error, while Newton follows a vein of pure gold.

\section{Truesdell, Essays in the History of Mechanics (1968), p. 88}

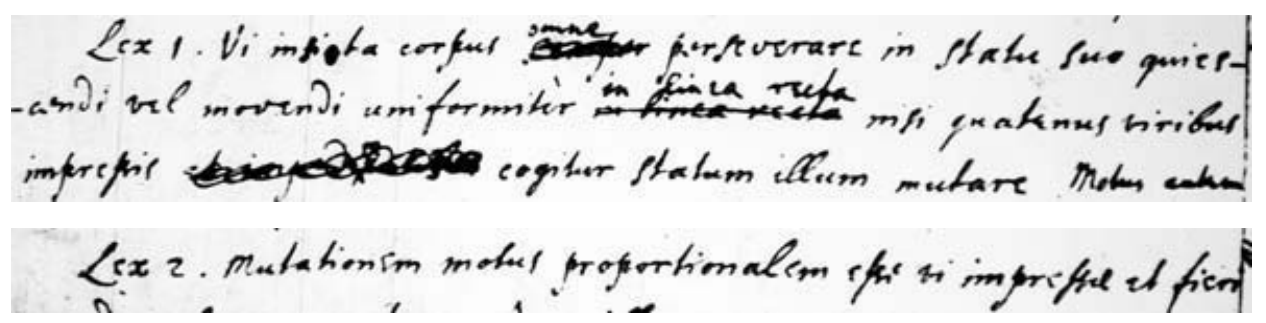

Figure 4.1. Newton's Lex 1 and Lex 2 from the manuscript Add. $3965^{7 a}$.

The first principles of motion fill many pages of axioms, theorems and discussions of the third 'day' of Galileo's Discorsi and many pages of beautiful prose in Barrow's lectures; for example: 'The following Axiom of Aristotle concerning Motion is famous .... He that is ignorant of Motion, must necessarily know nothing of Nature,' (Barrow 1670, p. 2), or 'You know the very trite Saying of St. Austin, ${ }^{7}$ If no one asks me, I know; but if any Person should require me to tell him, I cannot,' (Barrow 1670, p. 4). But Newton subsumes everything into three short and precise laws, the first two of which are as follows (see Figure 4.1).

Lex 1. Without force a body remains in uniform motion on a straight line.

Lex 2. The change of motion is proportional to the motive force impressed.

These laws were also expressed independently by Huygens (1673).

The basic idea is now displayed in Figure 4.2. Instead of thinking of the body moving on a curve $A B C D E F \ldots$ under the continuous influence of a force $F$ acting from the Sun, we let it move under Lex 1 from $A$ to $B$ during a time interval $\Delta t$ without force, and replace the forces acting by one force impulse of size $\Delta t \cdot F$ at the end of this step. If this force is directed towards the Sun, by Lex 2 the velocity which was in direction $A B$ will change to the

${ }^{6}$ With courtesy of Cambridge University Library.

${ }^{7}$ St. Augustine. 

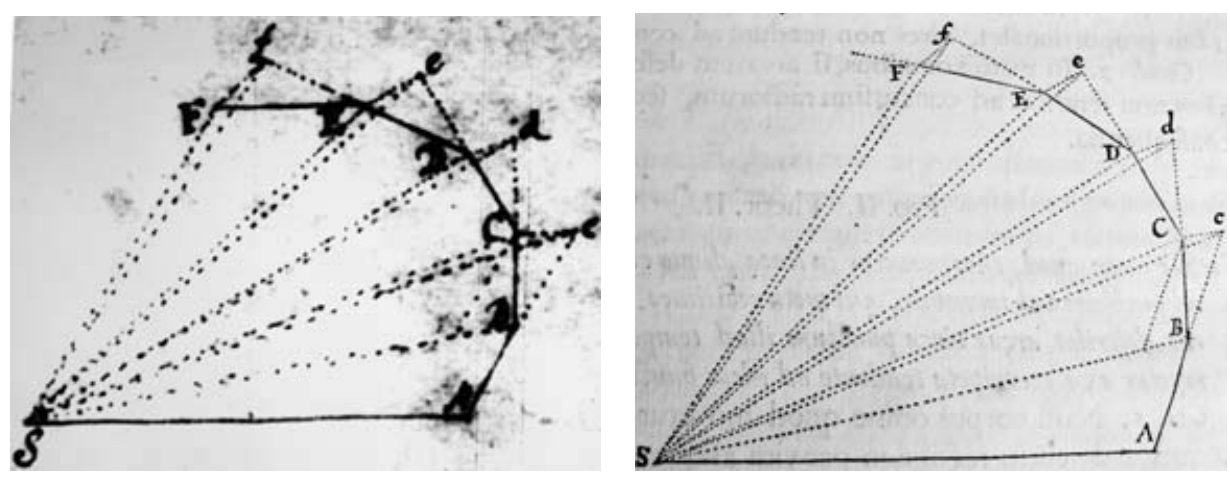

Figure 4.2. Newton's drawing of the symplectic Euler method for the proof of Kepler's Second Law; manuscript Add. 3965 ${ }^{7 a}$ (left), publication in the Principia (right).

direction $A V$ such that $B V S$ are aligned. So for the next time interval, the body will move from $B$ to $C$ such that $A B C V$ is a parallelogram.

The proof of Kepler's Second Law is now as follows (see Figure 4.3). If the force impulse at $B$ had not occurred, the body would have continued under Lex 1 until $c$ such that $A B=B c$. The triangles $S A B$ and $S B c$ have the same altitudes and the same bases. Hence, by Eucl. I.41, they have the same areas. Next, since the triangles $A B V$ and $B c C$ are the same, $c C$ will be parallel to $B V$, which, by hypothesis, is in the direction of $B S$. This means that the triangles $S B C$ and $S B C$ again have the same bases (which is $S B$ ) and the same altitudes, and thus the same areas. We conclude that $A B S$ has the same area as $B C S$, and if we continue like this, all triangles $A B S, B C S, C D S, D E S$, etc., will have the same areas.

Remark. If we recall the explanations of Section 1.4 above, we see that this proof of Newton uses precisely the symplectic Euler method, because the force impulse uses the force evaluated at the end of the interval. Here, this method is used inversely; the properties of the numerical solution are pulled
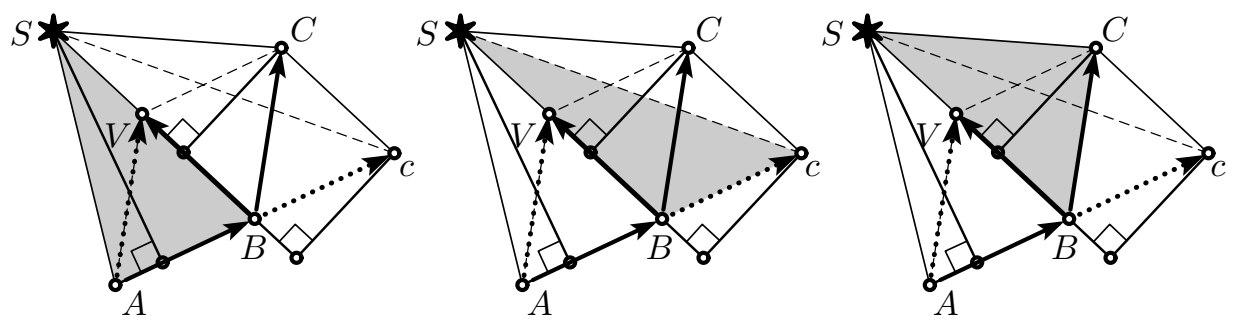

Figure 4.3. Eucl. I.41: All the triangles have the same area; Newton's proof of Kepler's Second Law. 
back to the continuous problem, and then constitute Kepler's Second Law. This became the 'Theorema 1' of the Principia and Kepler would surely have been very happy with this superbly elegant proof.

\subsection{Newton's discovery of the Law of Gravitation from Kepler's First and Second Laws}

... one of the most far-reaching generalizations of the human mind. While we are admiring the human mind, we should take some time off to stand in awe of a nature that could follow with such completeness and generality such an elegantly simple principle as the law of gravitation.

R. Feynman (1963); published in Six Easy Pieces (1994), p. 89

The next, and greater, challenge is to find out the quantity of this force. Two lemmas will give the answer.

Lemma 1. For a fixed time interval $\Delta t$, the force impulse is proportional to the distance $R Q$, where $R$ is on the tangent and $Q$ is on the orbit (see Figure 4.4, right).

Proof. Newton's motivation is shown in Figure 4.4 (left). Let our body move, under the continuous force, from $A$ to $D$. If there were no force, it would move to $B$ on the tangent; if it had no initial velocity, it would move to $C$. By a principle of superposition of forces and movements (which requires another lemma), we see that $B D=A C$, which is proportional, for a fixed $\Delta t$ and by Lex 2 , to the acting force. Huygens (1673) illustrated the same result with the picture shown in Figure 4.4 (centre).
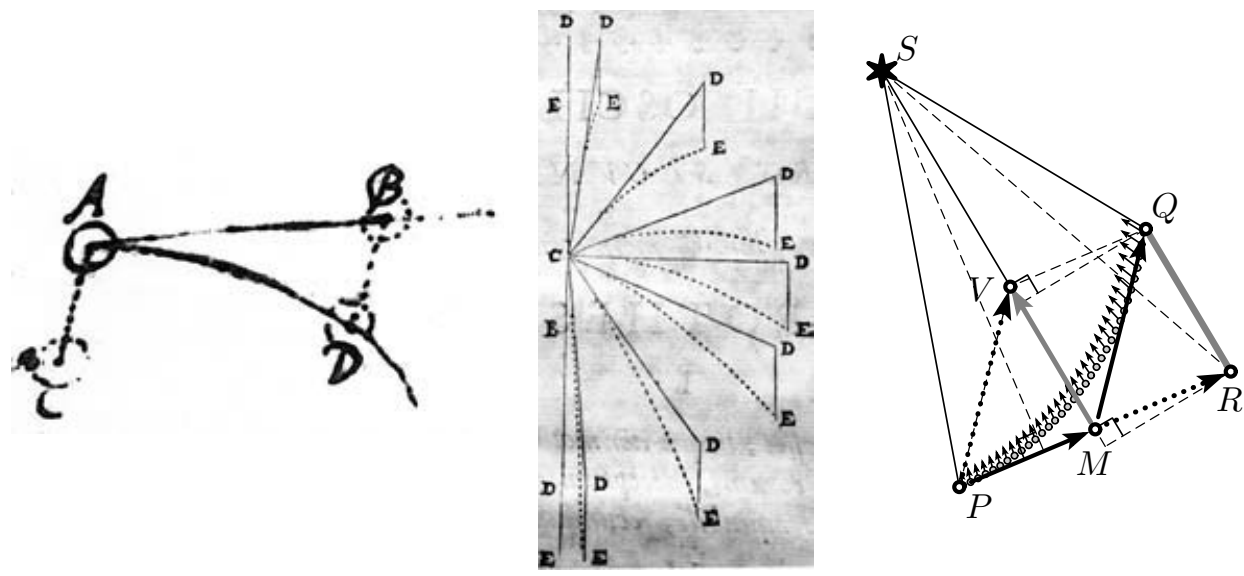

Figure 4.4. Proof of Lemma 1: Newton's manuscript Add. $3965^{6}$, from 1684 (left); Huygens, 1673 (centre); the Störmer-Verlet method (right). 
A third way of seeing this result is to place the force impulse $\Delta t \cdot F$ in the middle $M$ of the time interval (Figure 4.4, right). Our intuitive intelligence, and more numerical analysis, tell us that the resulting point $Q$ is very close to the orbit, and by parallel lines we see that the acting force, which is proportional to $M V$ by Lex 2 , is also proportional to $R Q$.

This last method bears the name Störmer-Verlet. It is also symplectic; many further properties are explained in Hairer, Lubich and Wanner (2003).

Lemma 2. Let an ellipse be given with focus $S$. Then there is a constant such that, for every $P$ on the ellipse, $Q$ on the ellipse close to $P, P R$ a tangent, RQS and PTS aligned and $T$ the orthogonal projection of $Q$ to $P S$ (see Figure 4.5), we have

$$
R Q \approx \text { Const } \cdot Q T^{2}
$$

Proof. Half of Newton's proof is displayed in Figure 4.6, where Lemma 2 is called 'Prob. 3'. Most readers, perhaps, would like some more explanations. The result is easier to see for circular motion (see Figure 4.7), where the formula $A T \cdot T P=Q T^{2}$ is known in geometry as the 'Theorem of the Altitudes', or, for experts, Eucl. II.14. Then, if $Q$ moves towards $P$,

$$
R Q \rightarrow T P=\frac{Q T^{2}}{A T} \approx \frac{Q T^{2}}{2 a} .
$$

In the general case we draw the 'diametri conjugata' $G P$ and $D K$. The latter is parallel to the tangent, to which we draw the parallel $Q X V$ (see Figure 4.6 as well as Figure 4.8). Now formula (4.2) has to be scaled by the halved lengths of these diameters, for which we use the letters $c$ and $d$ respectively, and we instead obtain

$$
V P \approx \frac{c}{2 d^{2}} \cdot Q V^{2}
$$

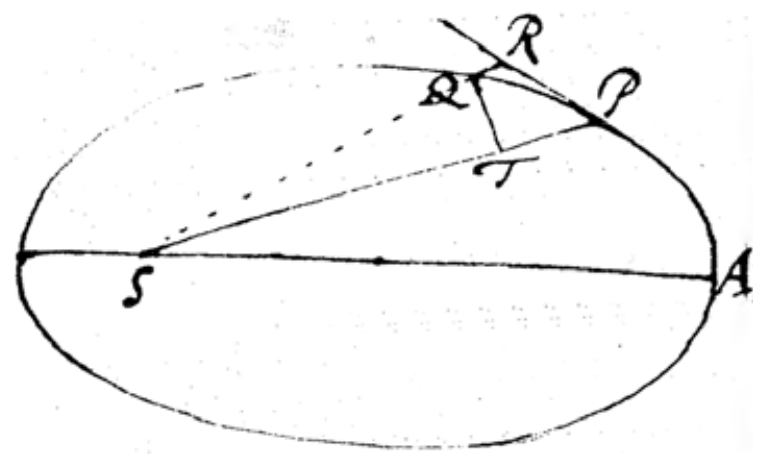

Figure 4.5. Newton's drawing for Lemma 2. 


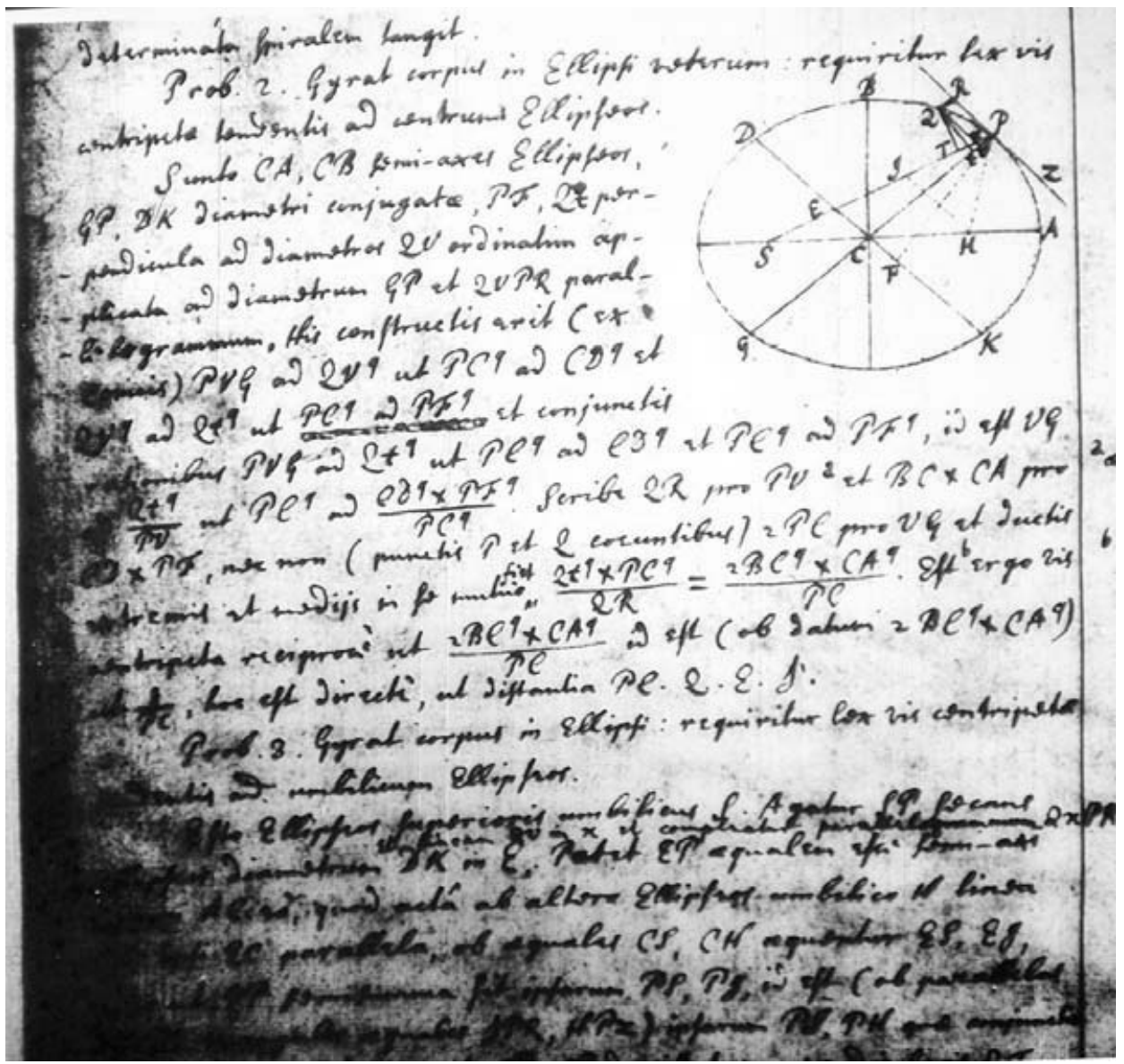

Figure 4.6. Proof of Lemma 2 in Newton's autograph Add. $3965^{6}$ (1684).
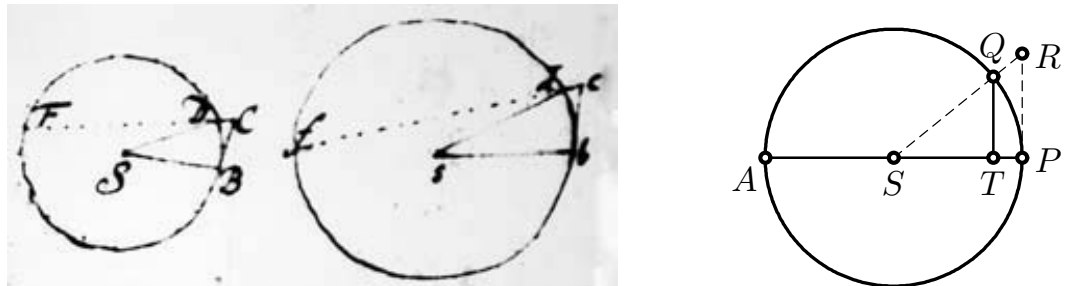

Figure 4.7. The proof of Lemma 2 for circular motion. 


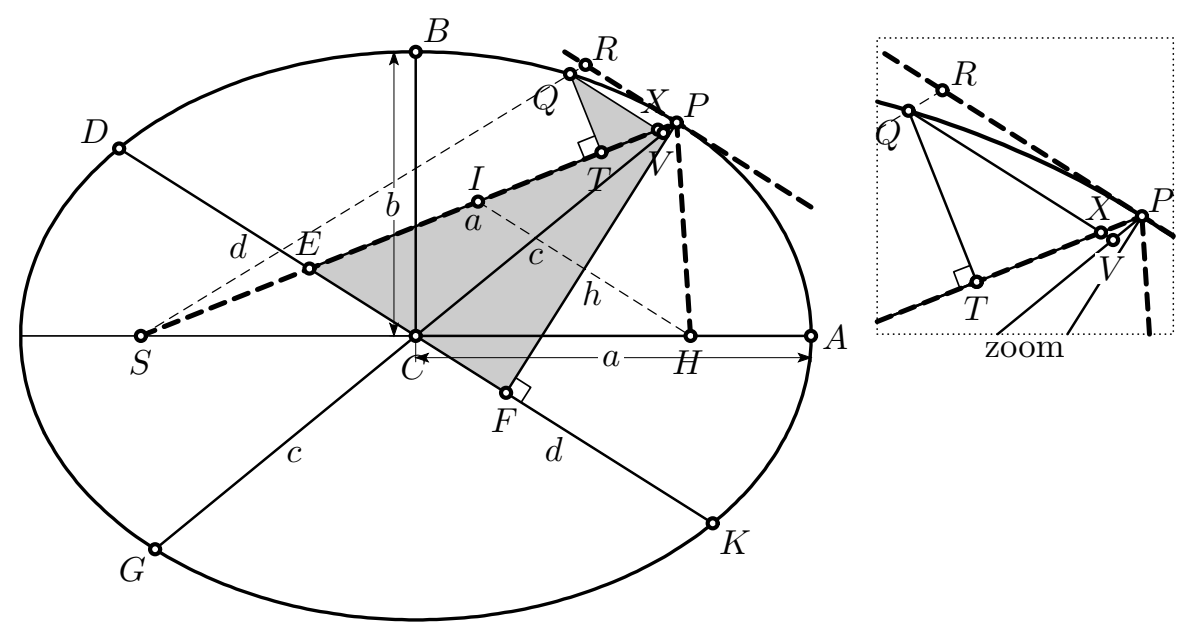

Figure 4.8. Modern illustration of Newton's proof of Lemma 2.

The next result we need is that $E P=a$, the major semi-axis. This follows from known theorems of Apollonius, i.e., that $S P+P H=2 a, S E=E I$ and $I P=P H$, but for Newton it was not an easy task (see the beginning of the proof of 'Prob. 3' in the lower part of Figure 4.6). This allows us to compute $V P$ from $X P \approx R Q$ and $Q V \approx Q X$ from $Q T$, by similar triangles $X V P \sim E C P$ as well as $Q T X \sim P F E$, giving

$$
V P \approx \frac{c}{a} \cdot R Q \quad \text { and } \quad Q V \approx \frac{a}{h} \cdot Q T .
$$

These two formulas inserted into (4.3) lead to

$$
R Q \approx \frac{a^{3}}{2 h^{2} d^{2}} \cdot Q T^{2}
$$

Our last difficulty is in understanding that $h d=a b$. This is another theorem of Apollonius (Apoll. VII.31) which Newton had to rediscover, and which states that parallelograms based on conjugate diameters of an ellipse all have the same area. Inserting this into (4.5), we finally obtain

$$
R Q \approx \frac{a}{2 b^{2}} \cdot Q T^{2}
$$

the desired result.

Theorem 1. (Proposition XI of the Principia) A body $P$, orbiting according to Kepler's First and Second Laws, is moving under the effect of a centripetal force, directed to the centre $S$, inversely proportional to the square of the distance. 

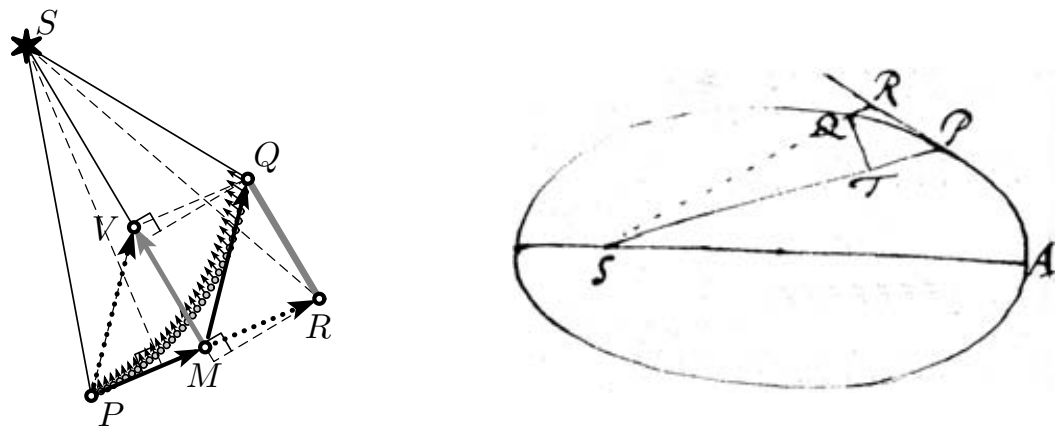

Force proportional to $R Q$ (Lemma 1) $\quad R Q$ proportional to $Q T^{2}$ (Lemma 2)

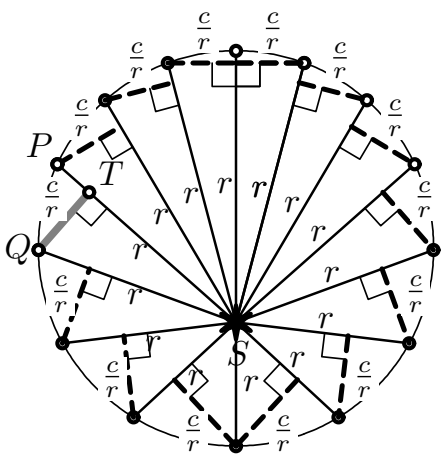

$Q T$ proportional to $\frac{1}{r}$ (Kepler 2)

Figure 4.9. The three steps of the proof of the Law of Gravitation.

The proof is in three steps, as shown in Figure 4.9, by combining Lemma 1 , Lemma 2 and Kepler's Second Law, which together lead to:

The force is proportional to $\frac{1}{r^{2}}$.

Another century later we arrive at Euler E112 (1749, p. 103), where the so-called 'Newton's equations'

$$
\text { I. } \frac{2 d d x}{d t^{2}}=\frac{\mathrm{X}}{\mathrm{M}} ; \text { II. } \frac{2 d d y}{d t^{2}}=\frac{\mathrm{Y}}{\mathrm{M}} ; \text { III. } \frac{2 d d z}{d t^{2}}=\frac{\mathrm{Z}}{\mathrm{M}}
$$

are published for the first time, and for which Newton's ideas are inverse numerical methods.

While physicists call these 'Newton's equations', they occur nowhere in the work of Newton or of anyone else prior to $1747 \ldots$ such is the universal ignorance of the true history of mechanics.

C. Truesdell, Essays in the History of Mechanics (1968), p. 117 


\section{Richard Feynman's elegant proof}

Pour voir présentement que cette courbe $A B C$... est toûjours une Section Conique, ainsi que M. Newton l'a supposé ... sans le démontrer; il y faut bien plus d'adresse:

(To see that the curve $A B C \ldots$ is always a conic section, which Newton supposed it to be, without proof, requires much more ability.)

Johann Bernoulli (1710); in Radelet-de Grave and Villaggio (2007)

... no calculus required, no differential equations, no conservation laws, no dynamics, no angular momentum, no constants of integration. This is Feynman at his best: reducing something seemingly big, complicated, and difficult to something small, simple, and easy.

B. Beckman (2006)

As we have just seen, in the Principia of 1687 Newton proved that a body moving around the Sun according to Kepler's First and Second Laws possesses the centrifugal force prescribed by the inverse square law. The reciprocal question, however, still remains open: Is every movement under a central inverse square force always an ellipse (or a conic)? Johann Bernoulli, in his usual sarcastic style, did not attribute to Newton enough ability ('adresse') to answer this question (see quotation). For other authors such as Arnol'd (1989), this result is clear from the uniqueness of the solutions. In any case, those who have seen one of the usual proofs in calculus know that the computations are not easy.

Fortunately, there is an elegant idea, in a 'lost lecture' of Richard Feynman, which D. L. and J. R. Goodstein (1996) discovered under inches of dust in Feynman's papers.

Feynman's idea. We see in Figure 5.1 the movement of our body moving under the inverse squares law represented with constant time steps $\Delta t$, so that the force impulses towards the Sun are proportional to $1 / r^{2}$. Let us now modify this picture (see Figure 5.2), not with constant time steps, but with constant angles $\Delta \phi$. Numerical analysts would say that we use another stepsize control. Now the areas of the triangles, which are all similar, become proportional to $r^{2}$ (this is Eucl. VI.19). Now, by Kepler's Second Law, whose proof is the same as for Lemma 1 above, all time steps $\Delta t$ become proportional to $r^{2}$. As a consequence, all force impulses $\Delta t \cdot F$ are:

(1) of constant length, and

(2) under an angle which changes constantly by $\Delta \phi$.

We now draw the velocities $\dot{P}$ in the velocity plane, called the hodograph. Because of Newton's Lex 2 and the two properties above, this hodograph 


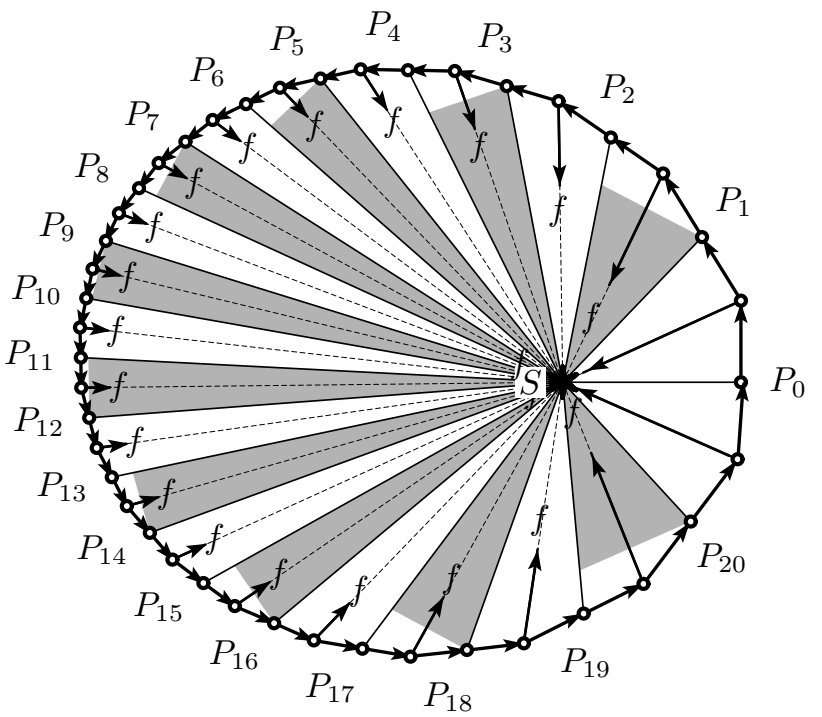

Figure 5.1. Planetary motion in constant time intervals.

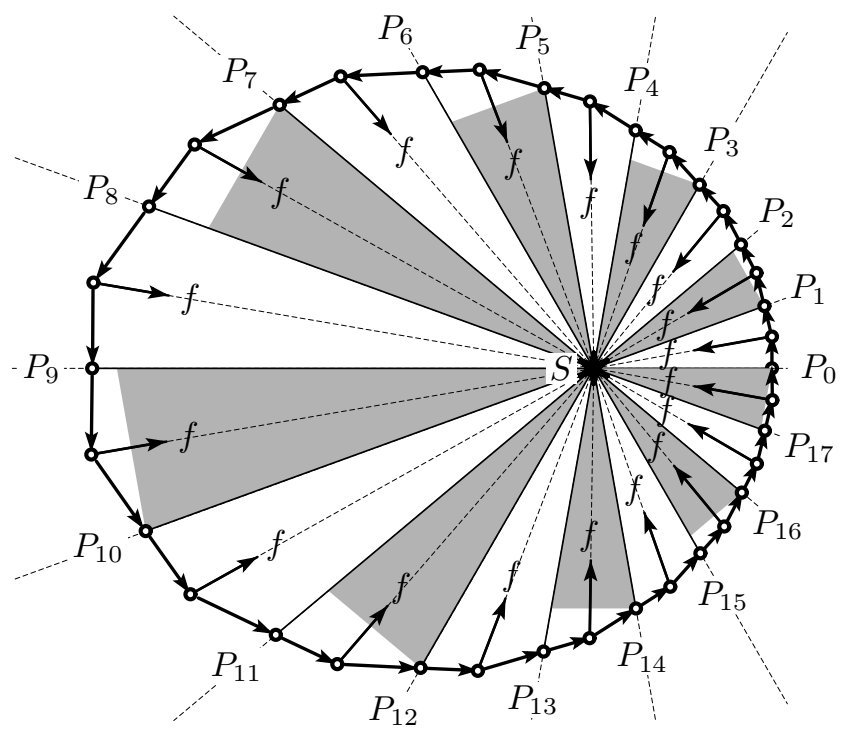

Figure 5.2. Planetary motion in constant angles. 

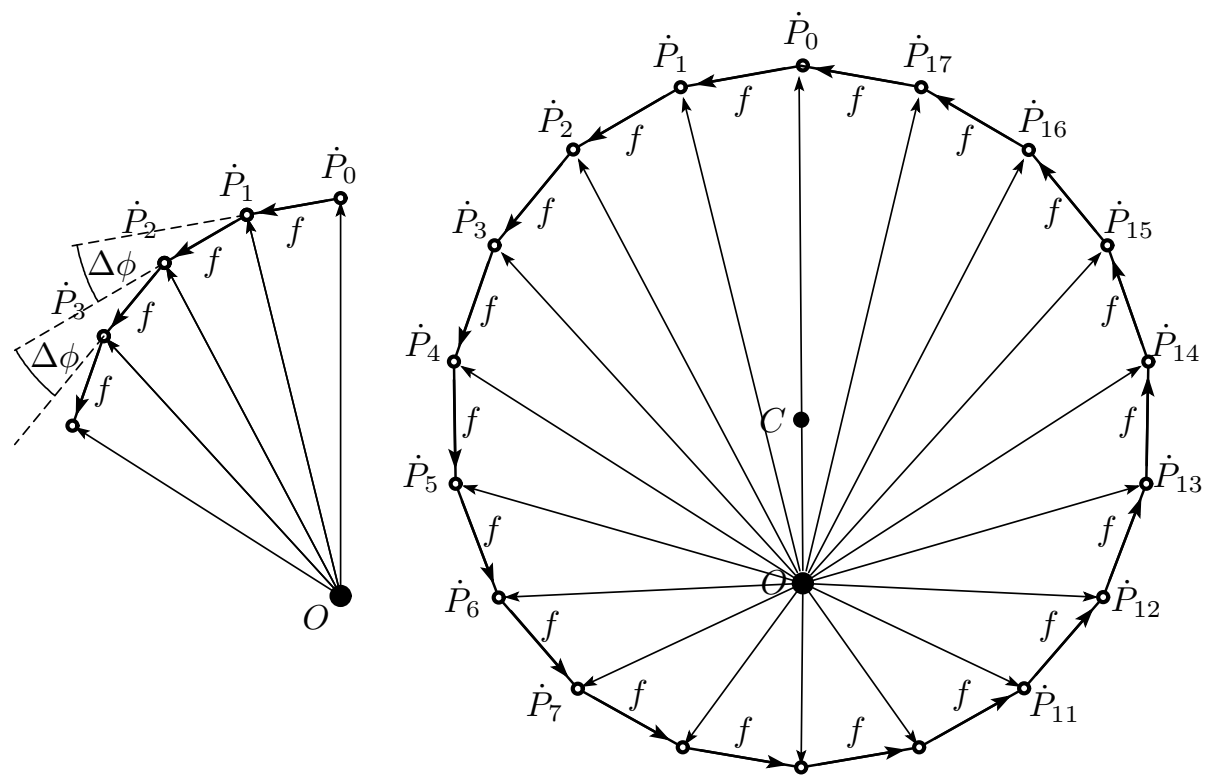

Figure 5.3. The hodograph for inverse square motion is a circle.

behaves like a dog which is dragged across the floor, pulled through the same distances each time, but in different directions. So the dog, starting from the velocity $\dot{P}_{0}$, which is in an upward direction with maximal speed, is first dragged to the left, later downwards, to $\dot{P}_{1}$, then to $\dot{P}_{2}$ and so on, describing the arc of a circle (Figure 5.3). The origin $O$, corresponding to speed 0, may lie in the circle (this corresponds to elliptic movement), or on the circle (parabolic), or outside the circle (hyperbolic movement).

Our last problem is to find a connection between the circle of Figure 5.3 and the orbit in Figure 5.2. Concerning this question, Feynman said 'I took a long time to find that,' and Beckman (2006) said 'Take a deep breath and look at the following.'

We know from geometry that if we have an ellipse with foci, say, $C$ and $O$, then for every point $P$ on the ellipse the sum of the distances satisfies $C P+P O=2 a$. This means, too, that the distance of $P$ from $O$ is the same as its distance from the circle centred at $C$ with radius $2 a$.

Well, let us have a second look at Figure 5.3: we see a circle centred at $C$ and a point $O$ inside this circle. This leads to the idea of considering the curve of points $P$ having the same distance from these two objects (see Figure 5.4). We know, as we just saw, that it is an ellipse. It is also known from geometry (Apoll. III.48), that the tangent at any point, say $\widetilde{P}_{3}$, reflects the ray $O \widetilde{P}_{3}$ to $C$, or, equivalently, that this tangent is orthogonal to $O \dot{P}_{3}$. On the contrary, in Figure 5.2 the tangent of the orbit is parallel to $O \dot{P}_{3}$. Furthermore, the angles in $C$, respectively $S$, move with the same constant 


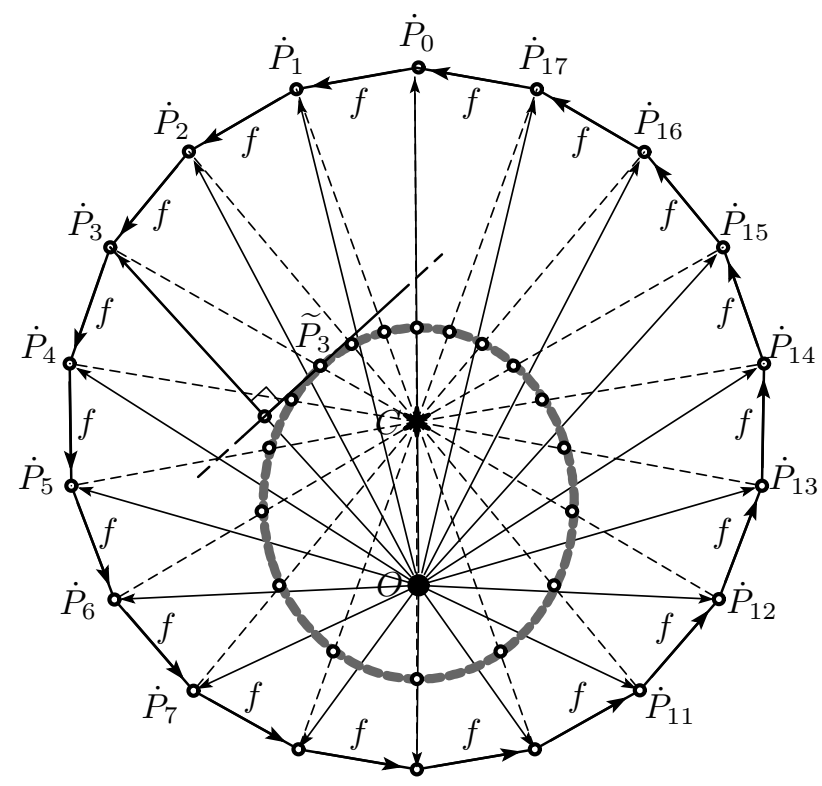

Figure 5.4. The hodograph with the ellipse of the same distance.

speed in both curves. Consequently, the orbit of Figure 5.2 is proportional to the ellipse of Figure 5.4, rotated by $90^{\circ}$. This concludes the proof.

Feynman comments on this beautiful proof as follows (lecture of March 13, 1964, 35th minute):

It is not easy to use the geometrical method to discover things. It is very difficult, but the elegance of the demonstrations after the discoveries are made is really very great. The power of the analytic method is that it is much easier to discover things and to prove things. But not in any degree of elegance. It's a lot of dirty paper, with $x$ 's and $y$ 's and crossed out cancellations and so on. [laughter]

Published in D. L. and J. R. Goodstein, Feynman's Lost Lecture (1996)

What about rigour? Many mathematicians would not consider the above to constitute rigorous proofs but merely nice illustrations. For example, the footnotes in D. T. Whiteside's edition of Newton's Mathematical Papers (2008), trying to render Newton's proofs rigorous, with all sorts of curved movements and estimations, are three times as long as Newton's original text. We numerical analysts know, however, that all the above formulas and pictures represent numerical methods, which are known to converge for $h \rightarrow 0$ to the corresponding solution, and for which properties of the numerical solution carry over to corresponding properties of the continuous model. So there is no need to announce all the time, as did, for example, Beckman (2006), that 'the argument is water tight'. 


\section{Acknowledgements}

It is my pleasure to express my thanks first of all to Ernst Hairer, from whom and with whom I had the privilege to learn numerical analysis over many decades, further to Christian Lubich, our co-author of the latest book of our trilogy on the numerical analysis of differential equations, to Philippe Henry, my co-author of a book in progress on Euler, to Alexander Ostermann, my co-author of a book in progress on geometry, to Alexei Shadrin, Cambridge, and Christian Aebi and Bernard Gisin, Geneva, for important hints on the literature, and to Glennis Starling, Acta Numerica's copy-editor, for excellent and extremely helpful support.

I also thank the Bibliothèque de Genève, as well as the Bibliothèque Georges de Rham in Geneva, and Cambridge University Library for permission to reproduce all the beautiful photographs from the original works in their possession. All illustrations from printed books in the Bibliothèque de Genève (BGE) are reproduced, with permission, from the specimen kept under catalogue numbers Ka337 (Euler E342), Rb1 (Leibniz), La163 (Fourier), Ra406 (Lagrange), Ka368 (Euler E65), Kb12 (Almagest), Kb31 (Brahe), Ka123 (Kepler, Astronomia Nova), Kb39 (Kepler, Harmonices Mundi), Kc216 (Galileo), Kb127 (Newton, Principia), Kc182 (Huygens) and Ra3 (Euler E112).

\section{REFERENCES}

V. I. Arnol'd (1989), Huygens and Barrow, Newton and Hooke: Pioneers in Mathematical Analysis and Catastrophe Theory from Evolvements to Quasicrystals, Nauka Moskva (1989). English translation: Birkhäuser (1990).

I. Barrow (1670), Geometrical Lectures: Explaining the Generation, Nature and Properties of Curve Lines, translated from the Latin edition (published 1670), revised, corrected and amended by Isaac Newton, edited by Edmund Stone, published in London (1735).

B. Beckman (2006), 'Feynman says: Newton implies Kepler, no calculus needed!' J. Symbolic Geom. 1, 57-72.

N. Copernicus (1543), De Revolutionibus Orbium Coelestium, published in Nuremberg. Original manuscript preserved in the Jagiellonian University Library, Kraków, Poland.

R. De Vogelaere (1956), Methods of integration which preserve the contact transformation property of the Hamiltonian equations. Report no. 4, Department of Mathematics, University of Notre Dame, IN.

L. Euler (E65 1744), Methodus Inveniendi Lineas Curvas Maximi Minimive Proprietate Gaudentes Sive Solutio Problematis Isoperimetrici Latissimo Sensu Accepti, published in Lausanne and Geneva. Reprinted in Opera Omnia, Ser. I, Vol. XXIV.

L. Euler (E112 1749), Recherches sur le Mouvement des Corps Célestes en Général, Vol. 3 of Mem. de l'Acad. des Sciences de Berlin, pp. 93-143. Reprinted in Opera Omnia, Ser. II, Vol. XXV, pp. 1-44. 
L. Euler (E301 1766), De Motu Corporis ad Duo Centra Virium Fixa Attracti, Vol. 10 of Novi Comm. Acad. Scient. Petropolitanae, pp. 207-242. Reprinted in Opera Omnia, Ser. II, Vol. VI, pp. 209-246.

L. Euler (E328 1767), De Motu Corporis ad Duo Centra Virium Fixa Attracti, Vol. 11 of Novi Comm. Acad. Scient. Petropolitanae, pp. 152-184. Reprinted in Opera Omnia, Ser. II, Vol. VI, pp. 247-273.

L. Euler (E342 1768), Institutiones Calculi Integralis, Vol. I, St. Petersburg. Reprinted in Opera Omnia, Ser. I, Vol. XI.

L. Euler (E366 1769), Institutiones Calculi Integralis, Vol. II, St. Petersburg. Reprinted in Opera Omnia, Ser. I, Vol. XII.

L. Euler (E385 1770), Institutiones Calculi Integralis, Vol. III, St. Petersburg. Reprinted in Opera Omnia, Ser. I, Vol. XIII.

R. Feynman (1967), The Character of Physical Law, MIT Press.

R. Feynman (1994), Six Easy Pieces: Essentials of Physics, Explained by its Most Brilliant Teacher, Perseus Books.

J. B. J. Fourier (1822): La Théorie Analytique de la Chaleur, Paris. A manuscript from 1807, Sur la Propagation de la Chaleur, had its publication refused 'due to lack of rigour'.

G. Galilei (1629), Les Méchaniques de Galilée, Mathématicien $\&$ Ingénieur du Duc de Florence, Traduites de l'Italien par le P. Marin Mersenne, 1629 manuscript published in Paris (1634). Critical edition by B. Rochod, Paris (1966).

G. Galilei (1638), Discorsi e Dimostrazioni Matematiche, Intorno à Due Nuove Scienze, Attenti Alla Mecanica $\&$ i Movimenti Locali, del Signor Galileo Galilei Linceo, Elsevier, Leiden. Critical edition by E. Giusti, Giulio Einaudi Editore, Torino (1990). German translation by A. von Oettingen, Ostwald's Klassiker, Leipzig (1890/91).

D. L. Goodstein and J. R. Goodstein (1996), Feynman's Lost Lecture: The Motion of Planets Around the Sun, Norton, New York.

E. Hairer, C. Lubich and G. Wanner (2003), Geometric numerical integration illustrated by the Störmer-Verlet method. In Vol. 12 of Acta Numerica, Cambridge University Press, pp. 399-450.

E. Hairer, C. Lubich and G. Wanner (2006), Geometric Numerical Integration: Structure-Preserving Algorithms for Ordinary Differential Equations, 2nd edn, Springer, Berlin.

E. Hairer, S. P. Nørsett and G. Wanner (1993), Solving Ordinary Differential Equations I: Nonstiff Problems, 2nd edn, Springer, Heidelberg.

C. Huygens (1673), Horologium Oscillatorium, Sive de Motu Pendulorum ad Horologia Aptato Demonstrationes Geometricae, published in Paris.

J. Kepler (1604), Ad Vitellionem Paralipomena, Quibus Astronomiae Pars Optica Traditur, Potissimum de Artificiosa Observatione et Aestimatione Diametrorum Deliquiorumque, Solis $\&$ Lunae cum Exemplis Insignium Eclipsium. Reprinted in Gesammelte Werke, Vol. 2.

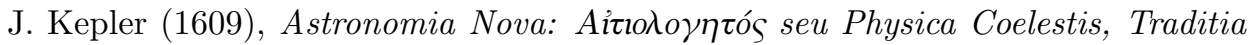
Commentariis de Motibus Stellae Martis, ex Observationibus G. V. Tychonis Brahe, published in Prague. Reprinted in Gesammelte Werke, Vol. 3, edited by M. Caspar (1937). French translation by J. Peyroux (1979). 
J. Kepler (1619), Harmonices Mundi, Libri V, published in Linz. Reprinted in Gesammelte Werke, Vol. 6, edited by M. Caspar (1940).

J. Kepler (1627), Tabulae Rudolphinae, published in Ulm. Reprinted in Gesammelte Werke vol. 10, edited by F. Hammer (1969).

J. L. de Lagrange (1759), 'Recherches sur la nature et la propagation du son', Miscellanea Taurinensia, Vol. I. Oeuvres, Vol. 1, pp. 39-148.

G. W. Leibniz (1693), Supplementum Geometriae Dimensoriae seu Generalissima Omnium Tetragonismorum Effectio per Motum: Similiterque Multiplex Constructio Linea ex Data Tangentium Conditione, published in Acta Eruditorum, Leipzig, pp. 385-392. German translation by G. Kowalewski, Leibniz über die Analysis des Unendlichen, No. 162 of Ostwalds Klassiker (1908), pp. 24-34.

S. Mandelbrote (2001), Footprints of the Lion, Cambridge University Library.

I. Newton (1684), several manuscripts preparing the Principia, preserved in Cambridge University Library, in particular manuscript Add. 3965 ${ }^{7 a}$ (1684).

I. Newton (1687), Philosophiae Naturalis Principia Mathematica, published in London. Russian translation with commentaries by A. N. Krylov (1936); reprinted (1989).

I. Newton (1713), Philosophiae Naturalis Principia Mathematica, 2nd edn.

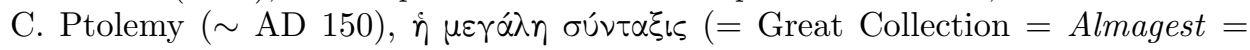
$A l \mu \varepsilon \gamma(\sigma \tau \eta)$. Latin translation by G. Peurbach and J. Regiomontanus, Epitoma Almagesti per Magistrum Georgium de Peurbach et eius Discipulum Magistrum Jo. de Künigsperg ..., completed 1462, printed 1496.

P. Radelet-de Grave and P. Villaggio, eds (2007), Die Werke von Johann I und Nicolaus II Bernoulli, Birkhäuser.

B. Russell (1931), The Scientific Outlook, Allen \& Unwin.

D. Tournès (2009), La Construction Tractionnelle des Équations Différentielles, Collection Sciences dans l'Histoire, Albert Blanchard, Paris.

C. Truesdell (1968), Essays in the History of Mechanics, Springer.

D. T. Whiteside, ed. (2008), The Mathematical Papers of Isaac Newton, Cambridge University Press. 